This is a postprint version of the following published document:

Rubin, M. B.; Rodríguez-Martínez, J.A. Influence of unobservable overstress in a rate-independent inelastic loading curve on dynamic necking of a bar, in: Mechanics of Materials. Special issue: IUTAM Symposium on Dynamic Instabilities in Solids, vol. 166, pp. 158-168, Jan. 2018

DOI: https://doi.org/10.1016/i.mechmat.2017.01.006

(C) 2017 Elsevier Ltd. All rights reserved.

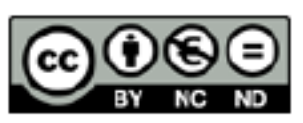

This work is licensed under a Creative Commons Attribution-NonCommercialNoDerivatives 4.0 International License. 


\title{
Influence of unobservable overstress in a rate-independent inelastic loading curve on dynamic necking of a bar
}

\author{
MB Rubin \\ Faculty of Mechanical Engineering \\ Technion - Israel Institute of Technology \\ 32000 Haifa, Israel \\ Email: mbrubin@tx.technion.ac.il
JA Rodríguez-Martínez
Department of Continuum Mechanics and Structural Analisis
University of Carlos III of Madrid
Avda. de la Universidad 30, 28911 Leganés
Madrid, Spain
Email: jarmarti@ing.uc3m.es

Submitted to the Mechanics of Materials

September 2016

Revised December 2016

Keywords: material instability; necking; rate-independent; smooth elastic-inelastic transition 


\begin{abstract}
Abstr act
A nonlinear rate-independent overstress model with a smooth elastic-inelastic transition is used to analyze instabilities during dynamic necking of a bar. In the simplified model the elastic strain $\varepsilon_{\mathrm{e}}$ determines the value of stress and the hardening parameter $\kappa$ determines the onset of inelasticity. These quantities $\left\{\varepsilon_{\mathrm{e}}, \kappa\right\}$ are obtained by integrating time evolution equations. The main and perhaps surprising result of this paper is that, based on the critical growth rate $\omega_{\mathrm{cr}}$ of a perturbation, two rate-independent materials with a smooth elastic-plastic transition due to overstress and nearly the same loading curve (elastic strain or stress versus total strain) can have different susceptibilities to tensile instabilities. Specifically, increase in overstress causes decreased material instability near the onset of the smooth elastic-inelastic transition and increased instability when the elastic strain approaches its saturated value. To the authors' knowledge, this new insight has not been reported in the literature.
\end{abstract}




\section{Introduction}

Eckart (1948) seems to be the first to have proposed evolution equations directly for elastic deformations to model the response of elastically isotropic elastic-inelastic solids. Similar equations were proposed by Leonov (1976) for polymeric liquids. Besseling (1966) proposed equations for elastically anisotropic solids. Another formulation for elastically anisotropic solids, which proposes evolution equations directly for elastic deformation measures, can be found in (Rubin, 1994). This later formulation is Eulerian and has the advantage that it removes unphysical arbitrariness of choices of the reference configuration, an intermediate configuration, a total deformation measure and a plastic deformation measure (Rubin, 2012).

Rate-independent models of materials with a smooth elastic-inelastic transition were developed for small deformations in (Lubliner et al., 1993; Einav, 2012) and a generalization for large deformations was presented in (Panoskaltsis, 2008). Recently Hollenstein et al. $(2013,2015)$ developed a generalized model for large deformation elastic-inelastic materials with an overstress term for the rate of inelastic deformation. Here, a special case of this theory is used to model rate-independent response of a material with a smooth elastic-inelastic transition.

The main features of this model can be explained by considering the one-dimensional theory. In particular, the model is formulated using an evolution equation for elastic strain which depends on the total rate of deformation and relaxation due to inelasticity. For this case the reference mass density $\rho_{0}$, strain energy function $\Sigma$ per unit mass, and the axial stress $\sigma$, and the rate of material dissipation $D$ are defined by

$$
\rho_{0} \Sigma=\frac{1}{2} \mathrm{E} \varepsilon_{\mathrm{e}}^{2}, \sigma=\rho_{0} \frac{\partial \Sigma}{\partial \varepsilon_{\mathrm{e}}}=\mathrm{E} \varepsilon_{\mathrm{e}}, \mathrm{D}=\sigma \dot{\varepsilon}-\rho_{0} \dot{\Sigma} \geq 0
$$


where $\mathrm{E}$ is the constant Young's modulus, $\varepsilon_{\mathrm{e}}$ is the axial elastic strain, $\dot{\varepsilon}$ is the total axial strain rate and a superposed $(\bullet)$ denotes material time differentiation. In the classical model the elastic strain is defined by the total strain $\varepsilon$ and a plastic strain $\varepsilon_{\mathrm{p}}$, which is determined by evolution equations, such that

$$
\varepsilon_{\mathrm{e}}=\varepsilon-\varepsilon_{\mathrm{p}}, \dot{\varepsilon}=\frac{\partial \mathrm{v}}{\partial \mathrm{x}}, \dot{\varepsilon}_{\mathrm{p}}=\Gamma\left(\frac{\sigma}{\mathrm{E}}\right), \Gamma \geq 0
$$

where $\partial \mathrm{v} / \partial \mathrm{x}$ is the velocity gradient and $\Gamma$ is a non-negative function that controls the rate of inelasticity. Motivated by Eckart (1948) who noted that the stress depends only on elastic strain $\varepsilon_{\mathrm{e}}$, it is possible to propose an evolution directly for elastic strain $\varepsilon_{\mathrm{e}}$ in the form

$$
\dot{\varepsilon}_{\mathrm{e}}=\frac{\partial \mathrm{v}}{\partial \mathrm{x}}-\Gamma \varepsilon_{\mathrm{e}}
$$

which can be obtained by differentiating (2a) and using (1b) and (2b). In particular, it is noted that (3) has an Eulerian form which does not depend on a definition of total strain $\varepsilon$ or plastic strain $\varepsilon_{\mathrm{p}}$. Moreover, using (1) and (3) it can be shown that the rate of material dissipation is given by

$$
\mathrm{D}=\Gamma \mathrm{E} \varepsilon_{\mathrm{e}}^{2} \geq 0
$$

which ensures that inelastic deformation is dissipative.

The three-dimensional large deformation model in (Hollenstein et al. 2013, 2015) is used here together with a Cosserat rod theory formulation (Rubin and RodríguezMartínez, 2014) to examine the influence of overstress in the smooth elastic-inelastic transition model on necking of a bar. The main and perhaps surprising result of this paper is that, based on the critical growth rate $\omega_{\mathrm{cr}}$ of a perturbation, two rate-independent 
materials with a smooth elastic-plastic transition due to overstress and nearly the same loading curve (elastic strain or stress versus total strain) can have different susceptibilities to tensile instabilities.

An outline of the paper is as follows. Section 2 records the basic equations of a model with a smooth elastic-inelastic transition which is based on integrating an evolution equation for elastic deformation, and Section 3 reviews a formulation of the necking problem based on Cosserat rod theory used in (Rubin and Rodríguez-Martínez, 2014). Section 4 develops the perturbation equations for linearized deformation superimposed on a nonlinear uniform solution and Section 5 considers the simplified case of no hardening. Section 6 discusses example problems and Section 7 presents conclusions. 
2. Basic equations for a smooth elastic-inelastic tran sition

For rate-independent response of an elastically isotropic material with a smooth elastic-inelastic transition, the total dilatation $\mathrm{J}$ and a unimodular (Flory, 1961), second order, symmetric, positive-definite tensorial measure $\mathrm{B}_{\mathrm{e}}^{\prime}$ of elastic distortional deformations are determined by integrating the evolution equations (Rubin and Attia, 1996)

$$
\dot{\mathrm{J}}=\mathrm{J} \mathrm{D} \cdot \mathrm{I}, \dot{\mathrm{B}}_{\mathrm{e}}^{\prime}=\mathrm{LB}_{\mathrm{e}}^{\prime}+\mathrm{B}_{\mathrm{e}}^{\prime} \mathrm{L}^{\mathrm{T}}-\frac{2}{3}(\mathrm{D} \cdot \mathrm{I}) \mathrm{B}_{\mathrm{e}}^{\prime}-\Gamma \mathrm{A}_{\mathrm{p}}
$$

In these equations, $\mathrm{L}$ is the gradient of the velocity $\mathrm{v}$ with respect to the present position $\mathrm{x}$ of a material point at time $\mathrm{t}, \mathrm{D}$ is the total deformation rate

$$
\mathrm{v}=\dot{\mathrm{x}}, \mathrm{L}=\partial \mathrm{v} / \partial \mathrm{x}, \mathrm{D}=\frac{1}{2}\left(\mathrm{~L}+\mathrm{L}^{\mathrm{T}}\right)
$$

the direction of inelastic deformation rate is characterized by $A_{p}$

$$
A_{p}=B_{e}^{\prime}-\left(\frac{3}{B_{e}^{\prime-1} \cdot I}\right) I
$$

the magnitude of inelastic deformation rate is controlled by the non-negative function $\{\Gamma \geq 0\}, \mathrm{I}$ is the second order unit tensor, $\mathrm{A} \cdot \mathrm{B}=\operatorname{tr}\left(\mathrm{AB}^{\mathrm{T}}\right)$ is the inner product between two second order tensor $\{\mathrm{A}, \mathrm{B}\}$ and a superposed $(\bullet)$ denotes material time differentiation.

For a rate-independent form of the model discussed in (Hollenstein et al., 2013) it is convenient to define a deviatoric elastic distortional strain tensor $\mathrm{g}_{\mathrm{e}}$, a scalar measure $\gamma_{\mathrm{e}}$ of elastic distortional strain

$$
\mathrm{g}_{\mathrm{e}}^{\prime \prime}=\frac{1}{2}\left[\mathrm{~B}_{\mathrm{e}}^{\prime}-\frac{1}{3}\left(\mathrm{~B}_{\mathrm{e}}^{\prime} \cdot \mathrm{I}\right) \mathrm{I}\right], \quad \gamma_{\mathrm{e}}=\sqrt{\frac{3}{2} \mathrm{~g}_{\mathrm{e}}^{\prime \prime} \cdot \mathrm{g}_{\mathrm{e}}^{\prime \prime}},
$$


and an effective total deformation rate $\dot{\varepsilon}_{\text {eff }}$

$$
\dot{\varepsilon}_{\text {eff }}=\sqrt{\frac{2}{3} \mathrm{D}^{\prime \prime} \cdot \mathrm{D}^{\prime \prime}}, \mathrm{D}^{\prime \prime}=\mathrm{D}-\frac{1}{3}(\mathrm{D} \cdot \mathrm{I}) \mathrm{I},
$$

where $\mathrm{D}^{\prime \prime}$ is the deviatoric part of D. Next, an overstress-type model is proposed in terms of a yield function $\mathrm{g}$, such that

$$
\mathrm{g}=1-\frac{\kappa \mathrm{f}}{\gamma_{\mathrm{e}}}, \mathrm{f}>0, \Gamma=\mathrm{b} \dot{\varepsilon}_{\mathrm{eff}}\langle\mathrm{g}\rangle, \mathrm{b} \geq 0
$$

with the hardening variable $\kappa$ being determined by integrating the evolution equation

$$
\dot{\kappa}=\mathrm{m} \Gamma\left(\kappa_{\mathrm{s}}-\kappa\right) \text {, }
$$

where $\mathrm{m}$ is a non-negative constant controlling the rate of hardening and $\kappa_{\mathrm{s}}$ is the saturated value of hardening. If the initial value $\kappa_{0}$ of hardening is smaller than $\kappa_{\mathrm{s}}$ then the material strengthens with increasing $\kappa$ during inelastic loading. However, by taking $\kappa_{0}$ greater than $\kappa_{\mathrm{s}}$ it is possible to model softening with decreasing $\kappa$. In these equations, the Macaulay brackets $\langle\mathrm{g}\rangle$ are defined by

$$
\langle\mathrm{g}\rangle=\max (0, \mathrm{~g})
$$

When $\Gamma$ vanishes, the solution of $(5 b) B_{e}^{\prime}$ equals the unimodular part of the left Cauchy-Green deformation tensor B so the model can characterize general isotropic elastic response as a special case. When $\Gamma$ does not vanish, the model characterizes an elastically isotropic material with inelastic response. Since $\Gamma$ is linear in the rate of deformation $\dot{\varepsilon}$, the evolution equations $(5 a, b)$ and (11) are homogeneous of order one in time so the material response is rate-independent. Yielding initiates when $\mathrm{g}=0$ with $\gamma_{\mathrm{e}}=\kappa \mathrm{f}$. At the onset of yielding the rate of inelasticity vanishes so the elastic-inelastic 
transition is smooth. The function $\mathrm{f}$, which is used to modify the yield strength due to the Bridgman (1952) effect for necking in a bar, is specified later [see (29)]. In addition, the constant $b$ controls the amount of overstress. Increased overstress (i.e. decrease in $b$ ) causes the curvature of the smooth elastic-inelastic transition to decrease. It will be shown that the value of $b$ influences the critical perturbation growth rate of the tensile instability being studied in this paper.

As a simple special case, consider a compressible Neo-Hookean form for the strain energy $\Sigma$ per unit mass given by

$$
\rho_{0} \Sigma=\frac{1}{2}\left[\mathrm{~K}(\mathrm{~J}-1)^{2}+\mu\left(\alpha_{1}-3\right)\right], \alpha_{1}=\mathrm{B}_{\mathrm{e}}^{\prime} \cdot \mathrm{I},
$$

where $\{K, \mu\}$ are the constant zero-stress bulk and shear modulus, respectively, and the reference mass density $\rho_{0}$ is related to the current mass density $\rho$ by the conservation of mass

$$
\rho J=\rho_{0} .
$$

Also, the rate of material dissipation $D$ is given by

$$
\mathrm{D}=\mathrm{T} \cdot \mathrm{D}-\rho \dot{\Sigma} \geq 0 .
$$

Then, using the procedures discussed in (Hollenstein et al., 2013) the Cauchy stress tensor $\mathrm{T}$ and the rate of material dissipation can be expressed in the forms

$$
\begin{gathered}
\mathrm{T}=-\mathrm{pI}+\mathrm{T}^{\prime \prime}, \mathrm{p}=-\rho_{0} \frac{\partial \Sigma}{\partial \mathrm{J}}=\mathrm{K}(1-\mathrm{J}), \mathrm{T}^{\prime \prime}=4 \mathrm{~J}^{-1} \rho_{0} \frac{\partial \Sigma}{\partial \alpha_{1}} \mathrm{ge}_{\mathrm{e}}^{\prime \prime}=2 \mathrm{~J}^{-1} \mu \mathrm{ge}_{\mathrm{e}}^{\prime \prime} \\
\mathrm{D}=\frac{1}{2} \mathrm{~J}^{-1} \mu \Gamma \mathrm{A}_{\mathrm{p}} \cdot \mathrm{I} \geq 0
\end{gathered}
$$


where $\mathrm{p}$ is the pressure and $\mathrm{T} "$ is the deviatoric part of $\mathrm{T}$. By expressing $\mathrm{B}_{\mathrm{e}}^{\prime} \cdot \mathrm{I}$ and $B_{e}^{\prime-1} \cdot I$ in terms of the eigenvalues of $B_{e}^{\prime}$ it can be shown that $A_{p} \cdot I$ is non-negative (Rubin and Attia, 1996). Also, the evolution equations (5) and (11) are integrated, subject to the initial conditions

$$
\mathrm{J}(0)=1, \mathrm{~B}_{\mathrm{e}}^{\prime}(0)=\mathrm{I}, \kappa(0)=\kappa_{0}>0,
$$

which characterize a stress-free state. 


\section{Cosserat $\mathrm{r}$ od theory formulation}

Figure 1 shows a sketch of a Cosserat rod that has a straight centerline and a circular cross-section having deformed radius $r=\phi B$. In its initial unstressed configuration, the rod has a uniform circular radius B and a material point is located by the axial coordinate Z. In its deformed configuration, $\mathrm{z}$ is the axial location of a material point and $\phi$ is the radial stretch

$$
\mathrm{z}=\mathrm{z}(\mathrm{Z}, \mathrm{t}), \phi=\phi(\mathrm{Z}, \mathrm{t})
$$

Within the context of the Cosserat rod model discussed in (Rubin and RodríguezMartínez, 2014) for axisymmetric deformations, the cross-section of the rod experiences homogeneous deformation.

For axisymmetric deformations the velocity gradient and the rate of deformation tensor are given by

$$
\mathrm{L}=\mathrm{D}=\frac{\dot{\phi}}{\phi}\left(\mathrm{e}_{1} \otimes \mathrm{e}_{1}+\mathrm{e}_{2} \otimes \mathrm{e}_{2}\right)+\frac{\dot{\lambda}}{\lambda}\left(\mathrm{e}_{3} \otimes \mathrm{e}_{3}\right), \lambda=\frac{\partial \mathrm{z}}{\partial \mathrm{Z}},
$$

where $\mathrm{e}_{\mathrm{i}}$ are fixed rectangular Cartesian base vectors, $\mathrm{a} \otimes \mathrm{b}$ denotes the tensor product between two vectors $\{a, b\}$ and $\lambda$ is the axial stretch. As in (Rubin and RodríguezMartínez, 2014), the material is approximated as being incompressible so the pressure $p$ in (16) is no longer given by a constitutive equation depending on $\mathrm{J}$ but instead is an arbitrary function of position and time and the deformation is isochoric with

$$
\mathrm{J}=\phi^{2} \lambda=1, \mathrm{D} \cdot \mathrm{I}=2\left(\frac{\dot{\phi}}{\phi}\right)+\frac{\dot{\lambda}}{\lambda}=0
$$

Using (9) and this result it can be shown that

$$
\dot{\varepsilon}_{\text {eff }}=\frac{|\dot{\lambda}|}{\lambda}
$$


Furthermore, for this deformation $\mathrm{B}_{\mathrm{e}}^{\prime}$ in (5b) and $\gamma_{\mathrm{e}}$ in (8) take the forms

$$
\mathrm{B}_{\mathrm{e}}^{\prime}=\frac{1}{\beta}\left(\mathrm{e}_{1} \otimes \mathrm{e}_{1}+\mathrm{e}_{2} \otimes \mathrm{e}_{2}\right)+\beta^{2}\left(\mathrm{e}_{3} \otimes \mathrm{e}_{3}\right), \quad \gamma_{\mathrm{e}}=\frac{\left|\beta^{3}-1\right|}{2 \beta},
$$

where $\beta$ is the elastic stretch in the axial direction and the evolution equation (5b) reduces to a single scalar equation of the form

$$
\frac{\dot{\beta}}{\beta}=\frac{\dot{\lambda}}{\lambda}-\mathrm{b}\left(\frac{\dot{\lambda} \mid}{\lambda}\right)\langle\mathrm{g}\rangle\left(\frac{\beta^{3}-1}{1+2 \beta^{3}}\right)
$$

Also, the rate of material dissipation (16) is given by

$$
\mathrm{D}=\mu \Gamma \frac{\left(\beta^{3}-1\right)^{2}}{\beta\left(1+2 \beta^{3}\right)} \geq 0 .
$$

Next, identifying $\mathrm{T}$ in (16) as the average Cauchy stress in (Rubin and RodríguezMartínez, 2014), the balance of linear momentum and director momentum take the forms

$$
\begin{aligned}
& \rho \ddot{z}=\frac{\partial}{\partial Z}\left(\phi^{2} \sigma\right), \rho y^{11} \ddot{\phi}=-\frac{1}{\phi}\left[-p+\frac{\mu\left(1-\beta^{3}\right)}{3 \beta}\right], \\
& \sigma=-p+\frac{2 \mu\left(\beta^{3}-1\right)}{3 \beta},
\end{aligned}
$$

where $\rho$ is constant mass density, $\sigma$ is the average axial stress, and the director inertia coefficient $y^{11}$, which characterizes inertia of cross-sectional deformations, is specified by (Rubin and Rodriguez-Martinez, 2014)

$$
\mathrm{y}^{11}=\frac{\mathrm{B}^{2}}{4} .
$$

Solving (25b) for the pressure $\mathrm{p}$ and substituting the result into (25a) yields the equation

$$
\rho \ddot{z}=\frac{\partial}{\partial Z}\left(\phi^{2} \sigma\right), \sigma=\frac{\mu\left(\beta^{3}-1\right)}{\beta}-\rho y^{11} \phi \ddot{\phi} .
$$

Furthermore, differentiating (27) with respect to $Z$ and using (19) and (20) yields 


$$
\ddot{\lambda}=\frac{\partial^{2}}{\partial Z^{2}}\left[\frac{\mu\left(\beta^{3}-1\right)}{\rho \beta \lambda}+\left(\frac{y^{11}}{2}\right) \lambda^{-3}\left(\ddot{\lambda}-\frac{3}{2} \lambda^{-1} \dot{\lambda}^{2}\right)\right] .
$$

Next, following the work in (Bridgman, 1952; Walsh, 1984; Fressengeas and Molinari, 1985; Zhou et al., 2006), the influence of the effect of the multiaxial stress state during necking can be approximated by specifying the function $f$ in (10) in the form

$$
\mathrm{f}=\left(1+\frac{1}{\theta}\right) \ln (1+\theta), \theta=\frac{1}{2} \mathrm{r} \frac{\partial^{2} \mathrm{r}}{\partial \mathrm{z}^{2}}=-\frac{\mathrm{B}^{2}}{4} \lambda^{-3 / 2} \frac{\partial}{\partial \mathrm{Z}}\left(\lambda^{-5 / 2} \frac{\partial \lambda}{\partial \mathrm{Z}}\right),
$$

where, with the help of (20), the current deformed radius $r$ of the rod is given by

$$
\mathrm{r}=\lambda^{-1 / 2} \mathrm{~B}
$$


4. Linearized deformation superimposed on a nonlinear uniform solution

In the following analysis it is convenient to define the normalized dimensionless variables $\{\zeta, \mathrm{T}\}$

$$
\zeta=\frac{\mathrm{Z}}{\mathrm{B}}, \mathrm{T}=\mathrm{Dt}, \mathrm{D}>0
$$

where $\mathrm{D}$ is a positive rate of stretch. Taking $\{\lambda, \beta, \kappa\}$ to be functions of $\{\zeta, \mathrm{T})$ the balance of linear momentum (28) and the evolution equations (23) and (11) can be written in the forms

$$
\begin{gathered}
\frac{\partial^{2} \lambda}{\partial \mathrm{T}^{2}}=\frac{\partial^{2}}{\partial \zeta^{2}}\left[\left(\frac{\mu}{\rho \mathrm{D}^{2} \mathrm{~B}^{2}}\right)\left(\frac{\beta^{3}-1}{\beta \lambda}\right)+\left(\frac{\mathrm{y}^{11}}{2 \mathrm{~B}^{2}}\right) \frac{1}{\lambda^{3}}\left\{\frac{\partial^{2} \lambda}{\partial \mathrm{T}^{2}}-\frac{3}{2}\left(\frac{1}{\lambda}\right)\left(\frac{\partial \lambda}{\partial \mathrm{T}}\right)^{2}\right\}\right] \\
\frac{\partial \beta}{\partial \mathrm{T}}=\left(\frac{\beta}{\lambda}\right) \frac{\partial \lambda}{\partial \mathrm{T}}-\mathrm{b}\left[\frac{\beta}{\lambda}\left|\frac{\partial \lambda}{\partial \mathrm{T}}\right|\right]\langle\mathrm{g}\rangle\left(\frac{\beta^{3}-1}{1+2 \beta^{3}}\right), \\
\frac{\partial \kappa}{\partial \mathrm{T}}=\mathrm{mb}\left[\frac{1}{\lambda}\left|\frac{\partial \lambda}{\partial \mathrm{T}}\right|\right]\langle\mathrm{g}\rangle\left(\kappa_{\mathrm{s}}-\kappa\right) .
\end{gathered}
$$

In particular, consider the nonlinear uniform solution of (32) with constant stretch rate for which

$$
\lambda=\lambda_{1}(\mathrm{~T}), \beta=\beta_{1}(\mathrm{~T}) \geq 1, \kappa=\kappa_{1}(\mathrm{~T}), \frac{\mathrm{d} \lambda}{\mathrm{dT}}= \pm 1
$$

where the functions $\left\{\beta_{1}, \kappa_{1}\right\}$ satisfy the equations

$$
\begin{gathered}
\left.\frac{\mathrm{d} \beta_{1}}{\mathrm{dT}}=\left(\frac{\beta_{1}}{\lambda_{1}}\right) \frac{\mathrm{d} \lambda_{1}}{\mathrm{dT}}-\mathrm{b}\left[\left(\frac{\beta_{1}}{\lambda_{1}}\right)\left|\frac{\mathrm{d} \lambda_{1}}{\mathrm{dT}}\right|\right]\left\langle\mathrm{g}_{1}\right\rangle\left(\frac{\beta_{1}^{3}-1}{1+2 \beta_{1}^{3}}\right)\right], \\
\frac{\mathrm{d} \kappa_{1}}{\mathrm{dT}}=\mathrm{m} \mathrm{b}\left[\left(\frac{1}{\lambda_{1}}\right)\left|\frac{\mathrm{d} \lambda_{1}}{\mathrm{dT}}\right|\right]\left\langle\mathrm{g}_{1}\right\rangle\left(\kappa_{\mathrm{s}}-\kappa_{1}\right),
\end{gathered}
$$

and with the help of (10) and (22), the yield function $g_{1}$ is given by 


$$
g_{1}=g_{1}(T)=1-\frac{2 \beta_{1} \kappa_{1}}{\beta_{1}^{3}-1}
$$

To analyze the stability of this solution, specify

$$
\lambda=\lambda_{1}(\mathrm{~T})+\eta_{1}(\zeta, T), \quad \beta=\beta_{1}(\mathrm{~T})+\eta_{2}(\zeta, T), \kappa=\kappa_{1}(\mathrm{~T})+\eta_{3}(\zeta, T)
$$

where $\eta_{i}$ are perturbations. Now, using the assumptions

$$
\frac{\partial \lambda}{\partial \mathrm{T}}>0, \mathrm{~g} \geq 0
$$

substituting (36) into the equations (32), using (33) and (35) and neglecting quadratic terms in $\eta_{i}$ and their derivatives yields the linearized equations (54) in the Appendix. Details of other developments described below are also recorded in the Appendix.

Next, consider the uniform solution of (32a) for $\lambda_{1}$ given by

$$
\lambda_{1}(\mathrm{~T})=1+\mathrm{T}
$$

Then, (34) are integrated numerically, subject to the initial conditions

$$
\beta_{1}(0)=1, \kappa_{1}(0)=\kappa_{0},
$$

to determine the values $\left\{\beta_{1}(\mathrm{~T}), \kappa_{1}(\mathrm{~T})\right\}$ at an arbitrary time $\mathrm{T}>0$. To ensure that the state at $\mathrm{T}$ is inelastic, the value $\mathrm{g}_{1}$ of the yield function should be positive with

$$
\mathrm{g}_{1}=1-\frac{2 \beta_{1} \kappa_{1}}{\beta_{1}^{3}-1}>1-\frac{2 \lambda_{1} \kappa_{0}}{\lambda_{1}^{3}-1}>0, \lambda_{1}>\beta_{1}>1, \kappa_{1}>\kappa_{0} .
$$

Moreover, using (20), (26) and (31), the axial stress $\sigma$ in (27) is given by

$$
\frac{\sigma}{3 \mu}=\frac{\beta_{1}^{3}-1}{3 \beta_{1}}+\left(\frac{\rho D^{2} B^{2}}{16 \mu}\right) \frac{1}{(1+T)^{3}}
$$


For short times the values of $\left\{\lambda_{1}, \beta_{1}, \kappa_{1}\right\}$ in (54) are approximated as constants and the solutions for the perturbations $\eta_{\mathrm{i}}$ are taken in the forms

$$
\eta_{i}=A_{i} \exp (\omega T) \cos (K \zeta)
$$

where $A_{i}$ are amplitudes, $\omega$ is a normalized frequency (perturbation growth rate) and $K$ is a normalized wave number. Then, with the help of (42) the equations (54) can be written in the matrix form

$$
\sum_{j=1}^{3} A_{i j}(\omega, K) A_{j}=0 \quad \text { for } i=1,2,3
$$

where the components of the matrix $\mathrm{A}_{\mathrm{ij}}$ are given in the Appendix by (56) and (57). It follows that non-trivial solutions of (43) require the determinant of $A_{i j}$ to vanish. This condition can be written as a quartic equation for $\omega$

$$
\mathrm{a}_{4} \omega^{4}+\mathrm{a}_{3} \omega^{3}+\mathrm{a}_{2} \omega^{2}+\mathrm{a}_{1} \omega+\mathrm{a}_{0}=0
$$

with all coefficients $a_{i}$ being functions of $\mathrm{K}$ given in the Appendix by (58). For the specified parameters, there is only one real positive value of $\omega$ which satisfies (44) for real positive values of $\mathrm{K}$. Now, the critical values $\left\{\mathrm{K}_{\mathrm{cr}}, \omega_{\mathrm{cr}}\right\}$ of $\{\mathrm{K}, \omega\}$ are determined by using this solution and satisfying the condition

$$
\frac{d \omega}{d K}=-\frac{\frac{d_{4}}{d K} \omega^{4}+\frac{d a_{3}}{d K} \omega^{3}+\frac{d a_{2}}{d K} \omega^{2}+\frac{d a_{1}}{d K} \omega+\frac{d_{0}}{d K}}{4 a_{4} \omega^{3}+3 a_{3} \omega^{2}+2 a_{2} \omega+a_{1}}=0 .
$$

The critical perturbation growth rate $\omega_{\mathrm{cr}}$ is a reference measure frequently used to assess the stability of materials (Guduru and Freund, 2002; Mercier and Molinari, 2003; Mercier 
et al., 2010; Zaera et al. 2014; Rodríguez-Martínez et al., 2015a; Rodríguez-Martínez et al., 2015b). Materials with large values of $\omega_{\mathrm{cr}}$ tend to develop instabilities. 
5. The simplified case of no hardening

In the absence of hardening $(\mathrm{m}=0), \kappa=\kappa_{1}=\kappa_{0}$ remains constant, the perturbation $\eta_{3}$ vanishes and the equations (43) reduce to

$$
\sum_{j=1}^{2} A_{i j}(\omega, K) A_{j}=0 \quad \text { for } i=1,2,
$$

with $\mathrm{A}_{\mathrm{ij}}$ given in the Appendix by (56) and (57). It then follows that the characteristic equation of (46) becomes

$$
a_{3} \omega^{3}+a_{2} \omega^{2}+a_{1} \omega+a_{0}=0
$$

with the coefficients given in the Appendix by (59). Furthermore, the critical values $\left\{\mathrm{K}_{\mathrm{cr}}\right.$, $\left.\omega_{\mathrm{cr}}\right\}$ of $\{\mathrm{K}, \omega\}$ are determined by the condition that

$$
\frac{\mathrm{d} \omega}{\mathrm{dK}}=-\frac{\frac{\mathrm{da}_{3}}{\mathrm{dK}} \omega^{3}+\frac{\mathrm{da}_{2}}{\mathrm{dK}} \omega^{2}+\frac{\mathrm{da}_{1}}{\mathrm{dK}} \omega+\frac{\mathrm{da}_{0}}{\mathrm{dK}}}{3 \mathrm{a}_{3} \omega^{2}+2 \mathrm{a}_{2} \omega+\mathrm{a}_{1}}=0
$$

together with the restriction that (47) must be satisfied. 


\section{Exam ple problems}

For the examples in this section, $\lambda_{1}$ is specified by (38) and the equations (34) are integrated numerically subject to the initial conditions (39) to determine the homogeneous values $\left\{\beta_{1}(\mathrm{~T}), \kappa_{1}(\mathrm{~T})\right\}$ at an arbitrary time $\mathrm{T}>0$. For each value of $\mathrm{T}$ associated with loading $\{\mathrm{d} \lambda / \mathrm{dT}=1\}$ and for which inelasticity is active with nonnegative values of $g_{1}$ in (40), the equations (47) and (48) can be solved for the critical values $\left\{\omega_{\mathrm{cr}}, \mathrm{K}_{\mathrm{cr}}\right\}$ of the perturbation growth rate $\omega$ and wave number $\mathrm{K}$ for the case of no hardening. Similarly, equations (44) and (45) can be solved to determine $\left\{\omega_{\mathrm{cr}}, \mathrm{K}_{\mathrm{cr}}\right\}$ for the case of hardening. Using this procedure, it is possible to determine values of $\{\lambda, \beta$, $\left.\omega_{\mathrm{cr}}, \mathrm{K}_{\mathrm{cr}}\right\}$ for all values of $\mathrm{T}$ for which $\mathrm{g}$ is non-negative and $\{\mathrm{d} \lambda / \mathrm{dT}=1\}$. Moreover, values of $\left\{\omega_{\mathrm{cr}}, \mathrm{K}_{\mathrm{cr}}\right\}$ are only presented for loading and inelastic response (37) where equations (43) and (46) are applicable. Also, for convenience the nominal total strain $\varepsilon$ and the nominal elastic strain $\varepsilon_{\mathrm{e}}$ are defined by

$$
\varepsilon=\lambda-1, \varepsilon_{\mathrm{e}}=\beta-1
$$

In addition, it is noted that for the parameters used in the following examples, the axial stress $\sigma$ given by (41) includes the term due to radial inertia effects. This term has negligible influence on the magnitude of the stress and for small elastic strain $\sigma /(3 \mu) \approx \varepsilon_{\mathrm{e}}$. However, radial inertia has been shown to influence the critical value of the perturbation growth rate $\omega_{\mathrm{cr}}$ (Rubin and Rodríguez-Martínez, 2014).

Note that, at the strain rates explored in this research, the increase of temperature in the material due to plastic deformation under adiabatic conditions may lead to meaningful 
strain softening which, in turn, promotes the formation of instabilities (Fressengeas and Molinari 1985, Zhou et al. 2006). Nevertheless, for the sake of simplicity and with the aim of focusing attention on the role played by overstress in the formation of necking instabilities, these thermal effects have been ignored in the analysis.

6.1 Monotonic loading with no har dening

Figure 2 shows the elastic strain $\varepsilon_{\mathrm{e}}$, axial stress $\sigma$, critical growth rate $\omega_{\mathrm{cr}}$ and wave number $\mathrm{K}_{\mathrm{cr}}$ versus nominal total strain $\varepsilon$ for

$$
\dot{\varepsilon}=10000[1 / \mathrm{s}], \mathrm{b}=500, \kappa=0.01, \mathrm{~m}=0 .
$$

These loading and material parameters are considered as a reference for the nonhardening materials analyzed in this section. The influence of these parameters on the response are explored in the following examples which vary one parameter while keeping the other reference parameters (50) fixed. The loading rate given by the nominal total strain rate $\dot{\varepsilon}$ is representative of dynamic experiments, like the rapid expansion of metallic rings (Zhang and Ravi-Chandar 2006) used in the characterization of engineering metals. The reference material parameters model the behavior of an idealized high-strength steel with large flow stress and negligible strain hardening. For all of the calculations presented in this paper, the additional material constants are specified by the shear modulus $\mu=75[\mathrm{GPa}]$ and the mass density $\rho=7850\left[\mathrm{~kg} / \mathrm{m}^{3}\right]$, which characterize steel. Also, the initial diameter of the rod is $B=1[\mathrm{~mm}]$, in agreement with typical ring expansion specimens (Zhang and Ravi-Chandar 2006).

For the $\varepsilon_{\mathrm{e}}-\varepsilon$ curve in Fig. 2a and the $\sigma-\varepsilon$ curve in Fig. 2b, low values of $\varepsilon$ correspond to the elastic regime in which $\left\{\varepsilon_{\mathrm{e}}, \sigma\right\}$ increase linearly with the nominal total strain. The 
linear relation between $\left\{\varepsilon_{\mathrm{e}}, \sigma\right\}$ and $\varepsilon$ ceases with the onset of yielding. Since $\Gamma$ vanishes at the onset of inelasticity, the elastic-inelastic transition is smooth so the onset of inelasticity is not visually detectable in the $\varepsilon_{\mathrm{e}}-\varepsilon$ and $\sigma-\varepsilon$ curves. This type of smooth transition has been observed in many engineering metals which do not show a definite yield point, see for example the experiments reported in a series of papers by NematNasser and co-workers (Nemat-Nasser et al., 2001; Nemat-Nasser and Guo, 2003, 2005; Guo and Nemat-Nasser, 2006). For continued loading the elastic strain $\varepsilon_{\mathrm{e}}$ and axial stress $\sigma$ attain their saturated values $\left\{\varepsilon_{\mathrm{es}}, \sigma_{\mathrm{s}}\right\}$ with total strain rate balanced by inelastic deformation rate. The value of $\kappa_{\mathrm{s}}$ associated with the saturated value $\varepsilon_{\mathrm{es}}$ is determined by substituting (35) into (34a), replacing $\left\{\lambda_{1}, \beta_{1}, \kappa_{1}\right\}$ by $\left\{1+\varepsilon, 1+\varepsilon_{\mathrm{e}}, \kappa_{\mathrm{s}}\right\}$, respectively, and limiting attention to loading with $\mathrm{d} \varepsilon / \mathrm{dT}>0$ to obtain the evolution equation

$$
\frac{\mathrm{d} \varepsilon_{\mathrm{e}}}{\mathrm{dT}}=\left(\frac{1+\varepsilon_{\mathrm{e}}}{1+\varepsilon}\right) \frac{\mathrm{d} \varepsilon}{\mathrm{dT}}\left[1-\mathrm{b}\left\langle 1-\frac{2\left(1+\varepsilon_{\mathrm{e}}\right) \kappa_{\mathrm{s}}}{\left(1+\varepsilon_{\mathrm{e}}\right)^{3}-1}\right\rangle\left\{\frac{\left(1+\varepsilon_{\mathrm{e}}\right)^{3}-1}{1+2\left(1+\varepsilon_{\mathrm{e}}\right)^{3}}\right\},\right.
$$

which is solved for the value of $\kappa_{\mathrm{s}}$ that causes $\mathrm{d} \varepsilon_{\mathrm{e}} / \mathrm{dT}$ to vanish with $\varepsilon_{\mathrm{e}}=\varepsilon_{\mathrm{es}}>0$ and $\mathrm{g}>0$

$$
\kappa_{\mathrm{s}}=\frac{1}{2\left(1+\varepsilon_{\mathrm{es}}\right)}\left[\mathrm{b}\left\{\left(1+\varepsilon_{\mathrm{es}}\right)^{3}-1\right\}-\left\{1+2\left(1+\varepsilon_{\mathrm{es}}\right)^{3}\right\}\right] .
$$

This value $\kappa_{\mathrm{s}}$ will be non-negative when

$$
\mathrm{b} \geq \frac{1+2\left(1+\varepsilon_{\mathrm{es}}\right)^{3}}{\left(1+\varepsilon_{\mathrm{es}}\right)^{3}-1} .
$$

In this regard, it is emphasized that although the $\varepsilon_{\mathrm{e}}-\varepsilon$ response in Fig. $2 \mathrm{a}$ and the $\sigma-\varepsilon$ response in Fig. $2 \mathrm{~b}$ suggest apparent hardening, they really exhibit the influence of the 
overstress since $\kappa$ is constant. From Figs. $2 \mathrm{a}$, b it is observed that $\sigma /(3 \mu) \approx \varepsilon_{\mathrm{e}}$, as stated above, so $\sigma-\varepsilon$ curves will not be presented in the remainder of this paper.

Figures $2 \mathrm{c}$,d show the $\omega_{\mathrm{cr}}-\varepsilon$ and $\mathrm{K}_{\mathrm{cr}}-\varepsilon$ curves, respectively, which are restricted to the region of inelastic loading that is consistent with the restrictions (37). The elasticinelastic transition leads to a rapid increase in $\left\{\omega_{\mathrm{cr}}, \mathrm{K}_{\mathrm{cr}}\right\}$ associated with the decrease in the slope of the $\varepsilon_{\mathrm{e}}-\varepsilon$ curve due to active inelasticity. The slight increase in $\left\{\omega_{\mathrm{cr}}, \mathrm{K}_{\mathrm{cr}}\right\}$ after $\varepsilon_{\mathrm{e}}$ has saturated is exclusively caused by the progressive thinning of the crosssection of the bar. Moreover, it is noted that an increase in $\mathrm{K}_{\mathrm{cr}}$ indicates a decrease in the critical wavelength.

Figure 3 examines the influence of the total strain rate $\dot{\varepsilon}$ for no hardening showing the critical growth rate $\omega_{\mathrm{cr}}$ versus nominal total strain $\varepsilon$ for three nominal strain rates $\dot{\varepsilon}=5000,10000,50000[1 / \mathrm{s}]$. While all of the $\omega_{\mathrm{cr}}{ }^{-\varepsilon}$ curves in Fig. 3 have similar shapes, increase in the strain rate causes a significant decrease of the critical growth rate $\omega_{\mathrm{cr}}$. Moreover, since the material response is rate-independent (i.e. the $\varepsilon_{\mathrm{e}}-\varepsilon$ curve is independent of $\dot{\varepsilon}$ ), this effect is due solely to the stabilizing effect of inertia, in agreement with the theoretical considerations reported by Fressengeas and Molinari $(1985,1994)$.

From (52) it can be shown that the saturated value $\varepsilon_{\mathrm{es}}$ of elastic strain $\varepsilon_{\mathrm{e}}$ increases with decrease in $b$ and increase in $\kappa_{\mathrm{s}}$. Figure 4 examines the influence of the overstress constant $\mathrm{b}$ for no hardening with different saturated values of $\varepsilon_{\mathrm{e}}$ showing the elastic strain $\varepsilon_{\mathrm{e}}$ and critical growth rate $\omega_{\mathrm{cr}}$ versus nominal total strain $\varepsilon$ for three values of $\mathrm{b}=125$, 
500 and 2500. Specifically, Fig. 4a shows that the curvature of the $\varepsilon_{\mathrm{e}}-\varepsilon$ curves increases and the saturated value $\varepsilon_{\mathrm{es}}$ of $\varepsilon_{\mathrm{e}}$ decreases with increasing values of $\mathrm{b}$. Furthermore, the onset of inelasticity is uninfluenced by changes in b and the extent (with respect to $\varepsilon$ ) of the transition region increases with decrease in $b$. Figure $4 b$ shows that decrease in $b$, associated with increase in the overstress, causes a stabilizing effect of decrease in the critical growth rate $\omega_{\mathrm{cr}}$ at the onset of the smooth elastic-inelastic transition and a destabilizing effect of increase in $\omega_{\mathrm{cr}}$ when the elastic strain $\varepsilon_{\mathrm{e}}$ saturates. These results are consistent with the nonlinear expression (52) relating the saturated values of the parameters.

Figure 5 examines the influence of $\kappa$ for no hardening showing the elastic strain $\varepsilon_{\mathrm{e}}$ and critical growth rate $\omega_{\text {cr }}$ versus nominal total strain $\varepsilon$ for $\dot{\varepsilon}=10000[1 / \mathrm{s}], \mathrm{b}=500$, $\mathrm{m}=0$ (no hardening) and three values of $\kappa=0.005,0.01$ and 0.02 . These results indicate that increase in $\kappa$ causes a delay in the onset of inelasticity and an increase in the saturated value of elastic strain $\varepsilon_{\mathrm{e}}$ (Fig. 5a), with an associated destabilizing effect of increase in $\omega_{\mathrm{cr}}$ in the saturated region (Fig. 5b).

To isolate the effect of the overstress parameter b, (52) is used to determine pairs of $\left\{\mathrm{b}, \kappa_{\mathrm{s}}\right\}$ that have the same saturated value $\varepsilon_{\mathrm{es}}$ of $\varepsilon_{\mathrm{e}}$, which is determined using the reference values $\left\{b=500, \kappa_{s}=0.01\right\}$. Figure 6 examines the influence of the overstress constant $\mathrm{b}$ for no hardening with the same saturated value $\varepsilon_{\mathrm{es}}$ of $\varepsilon_{\mathrm{e}}$ showing the elastic strain $\varepsilon_{\mathrm{e}}$, critical growth rate $\omega_{\mathrm{cr}}$ and critical wave number $\mathrm{K}_{\mathrm{cr}}$ versus nominal total strain $\varepsilon$ for the three combinations of $\left\{b, \kappa_{\mathrm{s}}\right\}$ given in Table 1. Figure 6a shows that these 
combinations of $\left\{\mathrm{b}, \kappa_{\mathrm{s}}\right\}$ produce the same saturated value of elastic strain $\varepsilon_{\mathrm{e}}$, as expected. Figures $6 \mathrm{~b}, \mathrm{c}$ show that the increase in $\kappa_{\mathrm{s}}$ delays the onset of inelasticity. Moreover, decrease in $b$ causes increased stability, exhibited by decrease in $\omega_{c r}$, at the onset of the elastic-inelastic transition region and increased instability, exhibited by increase in $\omega_{\mathrm{cr}}$, at the end of the transition region where $\varepsilon_{\mathrm{e}}$ becomes saturated. This emphasizes that the susceptibility of a material to trigger tensile instabilities at large strains, as indicated by the critical growth rate $\omega_{\mathrm{cr}}$, depends on the entire state of the material $\left\{\varepsilon_{\mathrm{e}}, \kappa\right\}$, which is not totally determined by the $\varepsilon_{\mathrm{e}}-\varepsilon$ or $\sigma-\varepsilon$ curves. This also indicates that the neck size, as characterized by the critical wave number $\mathrm{K}_{\mathrm{cr}}$, also depends on the entire state of the material.

6.2 Monotonic loading with hardening

To further understand the influence of the overstress parameter $b$ on material instability it is necessary to distinguish between the apparent hardening shown in Fig. 6a and actual hardening. To this end, Fig. 7 examines the influence of the saturated value $\kappa_{\mathrm{s}}$ of hardening showing the elastic strain $\varepsilon_{\mathrm{e}}$ and critical growth rate $\omega_{\mathrm{cr}}$ versus nominal total strain $\varepsilon$ for $\dot{\varepsilon}=10000[1 / \mathrm{s}], \mathrm{b}=500, \kappa_{0}=0.01, \mathrm{~m}=0.2$ and three values of $\kappa_{\mathrm{S}}(0.01$, $0.02,0.03)$. The case when $\kappa_{\mathrm{s}}=\kappa_{0}=0.01$ considers no hardening, whereas the other cases shown in Fig. 7a indicate hardening with the saturated value $\varepsilon_{\mathrm{es}}$ of elastic strain increasing with increasing $\kappa_{\mathrm{s}}$. For all cases, the onset of inelastic deformation occurs at the same point. Figure $7 \mathrm{~b}$ shows that increase in $\kappa_{\mathrm{S}}$ causes increased stability, exhibited 
by decrease in $\omega_{c r}$, at the onset of the elastic-inelastic transition region and increased instability, exhibited by increase in $\omega_{\mathrm{cr}}$, at the end of the transition region where $\varepsilon_{\mathrm{e}}$ tends to saturate.

Figure 8 examines the influence of the hardening rate $\mathrm{m}$ showing the elastic strain $\varepsilon_{\mathrm{e}}$, and critical growth rate $\omega_{\mathrm{cr}}$ versus the nominal total strain $\varepsilon$ for $\dot{\varepsilon}=10000[1 / \mathrm{s}], \mathrm{b}=500$, $\kappa_{0}=0.01, \kappa_{\mathrm{s}}=0.02$ and three values of $\mathrm{m}(0.1,0.2,0.8)$. As expected, Fig. 8a shows that the saturated value of elastic strain is unaffected by the value of $m$, which controls the rate at which $\varepsilon_{\mathrm{e}}$ approaches saturation. Also, Fig. $8 \mathrm{~b}$ indicates that increase in $\mathrm{m}$ causes increase in instability, exhibited by increase of $\omega_{\mathrm{cr}}$, at the onset of the smooth elasticinelastic transition and no increase in instability as $\varepsilon_{\mathrm{e}}$ approaches its saturated value.

Figure 9 shows the influence of the hardening rate $m$ for a small overstress $b=2500$ for a fixed value $\kappa_{\mathrm{s}}=0.012421$ and three values of $\left\{\kappa_{0}, \mathrm{~m}\right\}$ which yield the same saturated value $\varepsilon_{\mathrm{es}}$ of $\varepsilon_{\mathrm{e}}$. Figures $9 \mathrm{~b}, \mathrm{c}$ show that an increase in $\kappa_{0}$ (associated with an increase in m) causes a delay in the onset of inelasticity, increase in the instability and decrease in the wavelength at the onset of the smooth elastic-inelastic transition region with no increase in instability or decrease in wavelength as $\varepsilon_{\mathrm{e}}$ approaches its saturated value. To better isolate the influence of the overstress parameter $b$ on material instability, Fig. 10 examines the response for different values of $\left\{b, \kappa_{\mathrm{s}}, \mathrm{m}\right\}$ which yield nearly identical loading curves (Fig. 10a). Specifically, Figs. 10b,c clearly show that increased overstress (i.e. decreased b) causes a significant increase in material instability (i.e. 
increase in $\omega_{\mathrm{cr}}$ and decrease in the neck size associated with increase in $\mathrm{K}_{\mathrm{cr}}$ ) for the entire range of strains considered.

\subsection{Loading/unloading/reloading with hardening}

Figure 11 examines loading/unloading/reloading for a small overstress (with a large value of b) showing the elastic strain $\varepsilon_{\mathrm{e}}$ and critical growth rate $\omega_{\mathrm{cr}}$ versus the nominal total strain $\varepsilon$ for $\dot{\varepsilon}=10000[1 / \mathrm{s}], \mathrm{b}=500, \kappa_{0}=0.01, \kappa_{\mathrm{s}}=0.02$ and $\mathrm{m}=0.2$. The response for this value of $\mathrm{b}$ is typical of a metal with a rapid drop in $\varepsilon_{\mathrm{e}}$ at the onset of unloading and relatively high curvature of the elastic-inelastic transition during reloading. In addition, Fig. 12 examines loading/unloading/reloading for a large overstress (with a small value of b) showing the elastic strain $\varepsilon_{\mathrm{e}}$ and critical growth rate $\omega_{\mathrm{cr}}$ versus the nominal total strain $\varepsilon$ for $\dot{\varepsilon}=10000[1 / \mathrm{s}], \mathrm{b}=125, \kappa_{0}=0.00260, \kappa_{\mathrm{s}}=0.010860, \mathrm{~m}=$ 0.17. These material parameters produce nearly the same loading curve as those in Fig. 11a for small overstress, as indicated by the curve denoted by $(b=500)$ in Fig. 12a. Comparison of the results in Fig. 11a with Fig. 12a shows that increase in overstress (i.e. decrease in b) causes significant inelasticity at the onset of unloading and a significant reduction in the curvature of the elastic-inelastic transition during reloading (Fig. 12a). Moreover, comparison of the critical growth rates $\omega_{\mathrm{cr}}$ in Fig. $11 \mathrm{~b}$ and Fig. $12 \mathrm{~b}$ shows that increased overstress causes a significant increase in susceptibility to material instability. Similar comments could be made about the critical wave number $\mathrm{K}_{\mathrm{cr}}$. The large overstress associated with $b=125$ was considered to explore the influence of overstress on material instability but it produces unloading/reloading response (Fig. 12a) which has much smaller curvature in the elastic-inelastic transition than is typical of a metal. In this 
regard, the smaller increase in material instability shown in Fig. 10b for increase in overstress $(b=500)$ relative to $(b=2500)$ could be expected for metals. 


\section{Conclusions}

The linear perturbation analysis developed in this paper, using a rate-independent overstress model with a smooth elastic-inelastic transition based on an evolution equation for elastic strain, has led to new insights about the stability of metals subjected to dynamic tension. For a constant hardening variable, the monotonic loading curve (elastic strain versus total strain) shows apparent hardening due to the overstress of the smooth transition model, with increase in the elastic strain and stress to saturated values. By adjusting the material parameters controlling hardening it is possible to obtain nearly the same monotonic loading curves for materials with different values of the overstress parameter. Consequently, unlike for classical rate-independent plasticity theory which requires the yield function to remain non-positive, the monotonic loading curve for the overstress model does not characterize the entire state of the material. Additional information about unloading/reloading response is needed to determine the value of the overstress parameter. Numerical results of examples in this paper demonstrate that: (1) the apparent hardening introduced by overstress stabilizes material behavior at the onset of the smooth elastic-inelastic transition by slowing the rise of the critical perturbation growth rate; and (2) increase in overstress has a destabilizing influence on the critical growth rate when the material attains its saturated values. In particular, it has been shown that points on the same loading curve (elastic strain or axial stress versus total strain) can be at different material states due to overstress and thus can have different susceptibilities to tensile instabilities, as characterized by different values of the critical growth rate $\omega_{\text {cr }}$, the critical wave number $\mathrm{K}_{\mathrm{cr}}$ and the associated neck size. To the authors' knowledge, these new insights have not been reported in the literature. Moreover, this new insight 
suggests the need for future research to clarify differences in the predictions of the classical plasticity model and the smooth transition model for various modes of instabilities (e.g. shear and necking bands). In this regard, it is mentioned that Jabareen (2015) generalized this smooth transition model and calculated quasi-static necking of a bar. Furthermore, the constitutive model used in this paper can easily be generalized to include viscoplastic effects (e.g., Hollenstein et al., 2013) and thermal effects, which would enhance the capability of the model to reproduce available experimental data for different metals. Future work could use such a generalized model to assess the relative influences of viscous and thermal effects on the formation of necking instabilities.

In addition, it is noted that the Bridgman approximation (29) for necking was developed for elastic-perfectly plastic material response under quasi-static loading conditions. Here, this approximation has been used under dynamic loading conditions and for general cases which include actual hardening when $\kappa$ increases and apparent hardening when $\kappa$ remains constant. In this regard, it is noted that Vaz-Romero et al. (2016) have recently considered hardening materials and have compared results from a one-dimensional linear stability analysis of the kind developed in this paper with those for the three-dimensional approach developed by Mercier and Molinari (2003). Satisfactory agreement between the two models has been found, which confirms the validity of the Bridgman correction to describe the multiaxial stress state that develops inside a dynamically loaded necked section of a material with hardening. In addition, future research is planned to examine necking instabilities using the smooth transition model in finite element calculations which do not use the Bridgman approximation. 


\section{Acknowledgments}

JARM is indebted to the Ministerio de Economía y Competitividad de España (Projects EUIN2015-62556 and DPI2014-57989-P) for the financial support which permitted to conduct part of this work. The research leading to these results has received funding from the European Union's Horizon2020 Programme (Excellent Science, MarieSklodowska-Curie Actions) under REA grant agreement 675602 (Project OUTCOME). This research was also partially supported by MB Rubin's Gerard Swope Chair in Mechanics.

\section{References}

Besseling, J.F., 1966. A thermodynamic approach to rheology. Proc. of the IUTAM Symposium on irreversible aspects of continuum mechanics and transfer of physical characteristics in moving fluids, Vienna. (Eds. H. Parkus and L.I. Sedov), SpringerVerlag, Wein, 1968, 16-53.

Bridgman, P.W., 1952. Studies in large plastic flow and fracture, with special emphasis on the effects of hydrostatic pressure. vol. 1. New York: McGraw-Hill Book Company Inc; pp. 9-37.

Eckart, C., 1948. The thermodynamics of irreversible processes. IV. The theory of elasticity and anelasticity. Physical Review 73, 373-382.

Einav, I., 2012. The unification of hypoplastic and elasto-plastic theories. Int J Solids Struct. 49, 1305-1315.

Flory, P., 1961. Thermodynamic Relations For High Elastic Materials. Trans Faraday Soc. $57,829-838$. 
Fressengeas, C., Molinari, A., 1985. Inertia and thermal effects on the localization of plastic flow. Acta Metall. 33, 387-396.

Guduru, P.R., Freund, L.B., 2002. The dynamics of multiple neck formation and fragmentation in high rate extension of ductile materials. Int. J. Solids Struct. 39, $5615-5632$.

Guo, W.G., Nemat-Nasser, S., 2006. Flow stress of Nitronic-50 stainless steel over a wide range of strain rates and temperatures. Mechanics of Materials 38, 1090-1103.

Hollenstein, M., Jabareen, M. Rubin, M.B., 2013. Modeling a smooth elastic-inelastic transition with a strongly objective numerical integrator needing no iteration. Computational Mechanics 52, 649-667. Erratum, Computational Mechanics 55, 453.

Jabareen, M., 2015. Strongly objective numerical implementation and generalization of a unified large inelastic deformation model with a smooth elastic-inelastic transition. Int. J. Engng. Sci. 96, 46-67.

Leonov, A.I., 1976. Nonequilibrium thermodynamics and rheology of viscoelastic polymer media. Rheologica Acta 15, 85-98.

Lubliner, J., Taylor, R.L., Auricchio, F., 1993. A new model of generalized plasticity and its numerical implementation. Int. J Solids Struct. 30, 3171-3184.

Mercier, S., Molinari, A., 2003. Predictions of bifurcations and instabilities during dynamic extensions. Int. J Solids Struct. 40, 1995-2016.

Mercier, S., Granier, N., Molinari, A., Llorca, F., Buy, F., 2010. Multiple necking during the dynamic expansion of hemispherical metallic shells, from experiments to modelling. J. Mech. Phys. Solids 58, 955-982. 
Nemat-Nasser, S., Guo, W.G., Nesterenko, V.F., Indrakanti, S.S., Gu, Y.B., 2001. Dynamic response of conventional and hot isostatically pressed $\mathrm{Ti}-6 \mathrm{Al}-4 \mathrm{~V}$ alloys: experiments and modeling. Mechanics of Materials 33, 425-439.

Nemat-Nasser, S., Guo, W.G., 2003. Thermomechanical response of DH-36 structural steel over a wide range of strain rates and temperatures. Mechanics of Materials 35, $1023-1047$.

Nemat-Nasser, S., Guo, W.G., 2005. Thermomechanical response of HSLA-65 steel plates: experiments and modeling. Mechanics of Materials 37, 379-405.

Panoskaltsis, V.P., Polymenakos, L.C., Soldatos, D., 2008. On large deformation generalized plasticity. J. Mech. Mater. Struct. 3, 441-457.

Rodríguez-Martínez, J.A., Vadillo, G., Zaera, R., Fernández-Sáez, J., Rittel, D., 2015a. An analysis of microstructural and thermal softening effects in dynamic necking. Mechanics of Materials 80B, 298-310.

Rodríguez-Martínez, J.A., Vadillo, G., Rittel, D., Zaera, R., Fernández-Sáez, J., 2015b. Dynamic recrystallization and adiabatic shear localization. Mechanics of Materials $81,41-55$

Rubin, M.B., 1994. Plasticity theory formulated in terms of physically based microstructural variables - Part I: Theory. Int. J. Solids Struct. 31, 2615-2634.

Rubin, M.B., 2012. Removal of unphysical arbitrariness in constitutive equations for elastically anisotropic nonlinear elastic-viscoplastic solids. Int. J. Engng. Sci. 53, 3845.

Rubin, M.B., Attia, A.V., 1996. Calculation of hyperelastic response of finitely deformed elastic-viscoplastic materials. Int. J. Numerical. Meth. Engng. 39, 309-320. 
Rubin, M.B., Rodríguez-Martínez, J.A., 2014. The effect of radial inertia on flow localization in ductile rods subjected to dynamic extension. Int. J. Impact Engng. 69, $157-164$.

Vaz-Romero, A., Molinari, A., Rodríguez-Martínez J.A., Mercier, S., 2016. Multiple necking pattern in nonlinear elastic bars subjected to dynamic stretching: roles of defects and inertia. Submitted for publication.

Walsh, J.M., 1984. Plastic instability and particulation in stretching metal jets. J. Appl. Phys. 56, 1997-2006.

Zaera, R., Rodríguez-Martínez, J.A., Vadillo, G., Fernández-Sáez, J., 2014. Dynamic necking in materials with strain induced martensitic transformation. J. of the Mech. and Physics of Solids 64, 316-337.

Zhang, H., Ravi-Chandar, K., 2006. On the dynamics of necking and fragmentation - I. Real-time and post-mortem observations in Al 6061-O. Int. J. of Fracture 142, 183217.

Zhou, F., Molinari, J.F., Ramesh, K.T., 2006. An elasto-visco-plastic analysis of ductile expanding ring. Int. J. Impact Engng. 33, 880-891. 
Appendix: Details of the linear ized equations

Using the assumptions (37), substituting (36) into the equations (32), using (33) and (35) and neglecting quadratic terms in $\eta_{i}$ and their derivatives yields the linearized equations

$$
\begin{aligned}
& \frac{\partial^{2} \eta_{1}}{\partial \mathrm{T}^{2}}=\left[-\left(\frac{\mu}{\rho \mathrm{D}^{2} \mathrm{~B}^{2}}\right)\left(\frac{1}{\lambda_{1}^{2}}\right)\left(\frac{\beta_{1}^{3}-1}{\beta_{1}}\right)+6\left(\frac{\mathrm{y}^{11}}{2 \mathrm{~B}^{2}}\right)\left(\frac{1}{\lambda_{1}^{5}}\right)\right] \frac{\partial^{2} \eta_{1}}{\partial \zeta^{2}}+\left[\left(\frac{\mathrm{y}^{11}}{2 \mathrm{~B}^{2}}\right)\left(\frac{1}{\lambda_{1}^{3}}\right)\right] \frac{\partial^{4} \eta_{1}}{\partial \mathrm{T}^{2} \partial \zeta^{2}} \\
& -\left[3\left(\frac{\mathrm{y}^{11}}{2 \mathrm{~B}^{2}}\right)\left(\frac{1}{\lambda_{1}^{4}}\right)\right] \frac{\partial^{3} \eta_{1}}{\partial \mathrm{T} \partial \zeta^{2}}+\left[\left(\frac{\mu}{\rho \mathrm{D}^{2} \mathrm{~B}^{2}}\right)\left(\frac{1}{\lambda_{1}}\right)\left(\frac{1+2 \beta_{1}^{3}}{\beta_{1}^{2}}\right)\right] \frac{\partial^{2} \eta_{2}}{\partial \zeta^{2}} . \\
& \frac{\partial \eta_{2}}{\partial \mathrm{T}}=-\left(\frac{\beta_{1}}{\lambda_{1}^{2}}\right)\left[1-\operatorname{bg}_{1}\left(\frac{\beta_{1}^{3}-1}{1+2 \beta_{1}^{3}}\right)\right] \eta_{1}+\left(\frac{\beta_{1}}{\lambda_{1}}\right)\left[1-\operatorname{bg}_{1}\left(\frac{\beta_{1}^{3}-1}{1+2 \beta_{1}^{3}}\right)\right] \frac{\partial \eta_{1}}{\partial \mathrm{T}} \\
& -\left[\frac{\mathrm{b} \kappa_{1}}{4}\left(\frac{1}{\lambda_{1}^{5}}\right)\left(\frac{\beta_{1}^{2}}{1+2 \beta_{1}^{3}}\right)\right] \frac{\partial^{2} \eta_{1}}{\partial \zeta^{2}} \\
& +\left(\frac{1}{\lambda_{1}}\right)\left[1-\operatorname{bg}_{1}\left(\frac{\beta_{1}^{3}-1}{1+2 \beta_{1}^{3}}\right)-\operatorname{bg}_{1}\left\{\frac{9 \beta_{1}^{3}}{\left(1+2 \beta_{1}^{3}\right)^{2}}\right\}-2 \mathrm{~b}_{1}\left(\frac{\beta_{1}}{\beta_{1}^{3}-1}\right)\right] \eta_{2}+\left[2 \mathrm{~b}\left(\frac{1}{\lambda_{1}}\right)\left(\frac{\beta_{1}^{2}}{1+2 \beta_{1}^{3}}\right)\right] \eta_{3}, \\
& \frac{\partial \eta_{3}}{\partial \mathrm{T}}=-\mathrm{mb}\left[\left(\frac{1}{\lambda_{1}^{2}}\right) \mathrm{g}_{1}\left(\kappa_{\mathrm{s}}-\kappa_{1}\right)\right] \eta_{1}+\mathrm{mb}\left[\left(\frac{1}{\lambda_{1}}\right) \mathrm{g}_{1}\left(\kappa_{\mathrm{s}}-\kappa_{1}\right)\right] \frac{\partial \eta_{1}}{\partial \mathrm{T}} \\
& +\operatorname{mb}\left[\frac{1}{4}\left(\frac{1}{\lambda_{1}^{5}}\right)\left(\frac{\beta_{1}}{\beta_{1}^{3}-1}\right) \kappa_{1}\left(\kappa_{\mathrm{s}}-\kappa_{1}\right)\right] \frac{\partial^{2} \eta_{1}}{\partial \zeta^{2}}+\mathrm{mb}\left[2\left(\frac{1}{\lambda_{1}}\right)\left\{\frac{1+2 \beta_{1}^{3}}{\left(\beta_{1}^{3}-1\right)^{2}}\right\} \kappa_{1}\left(\kappa_{\mathrm{s}}-\kappa_{1}\right)\right] \eta_{2} \\
& -m b\left(\frac{1}{\lambda_{1}}\right)\left[g_{1}+2\left(\frac{\beta_{1}}{\beta_{1}^{3}-1}\right)\left(\kappa_{\mathrm{s}}-\kappa_{1}\right)\right] \eta_{3} .
\end{aligned}
$$

In these expressions, use has been made of the first order linearization

$$
g=g_{1}+\frac{1}{4 \lambda_{1}^{4}}\left(\frac{\beta_{1} \kappa_{1}}{\beta_{1}^{3}-1}\right) \frac{\partial^{2} \eta_{1}}{\partial \zeta^{2}}+2\left\{\frac{\left(1+2 \beta_{1}^{3}\right) \kappa_{1}}{\left(\beta_{1}^{3}-1\right)^{2}}\right\} \eta_{2}-2\left(\frac{\beta_{1}}{\beta_{1}^{3}-1}\right) \eta_{3} .
$$


Next, with the help of (42), the equations (54) can be written in the matrix form (43) where the components of the matrix $\mathrm{A}_{\mathrm{ij}}$ are given by

$$
\begin{gathered}
\mathrm{A}_{11}=\mathrm{a}_{11}(\mathrm{~K})+\mathrm{b}_{11}(\mathrm{~K}) \omega+\mathrm{c}_{11}(\mathrm{~K}) \omega^{2}, \mathrm{~A}_{12}=\mathrm{a}_{12}(\mathrm{~K}), \mathrm{A}_{13}=0, \\
\mathrm{~A}_{21}=\mathrm{a}_{21}(\mathrm{~K})+\mathrm{b}_{21} \omega, \mathrm{A}_{22}=\mathrm{a}_{22}+\mathrm{b}_{22} \omega, \mathrm{A}_{23}=\mathrm{a}_{23}, \\
\mathrm{~A}_{31}=\mathrm{a}_{31}(\mathrm{~K})+\mathrm{b}_{31} \omega, \mathrm{A}_{32}=\mathrm{a}_{32}, \mathrm{~A}_{33}=\mathrm{a}_{33}+\mathrm{b}_{33} \omega,
\end{gathered}
$$

and the coefficients are

$$
\begin{gathered}
\mathrm{a}_{11}=\left[\left(\frac{\mu}{\rho \mathrm{D}^{2} \mathrm{~B}^{2}}\right)\left(\frac{1}{\lambda_{1}^{2}}\right)\left(\frac{\beta_{1}^{3}-1}{\beta_{1}}\right)-6\left(\frac{\mathrm{y}^{11}}{2 \mathrm{~B}^{2}}\right)\left(\frac{1}{\lambda_{1}^{5}}\right)\right] \mathrm{K}^{2}, \mathrm{~b}_{11}=3\left(\frac{\mathrm{y}^{11}}{2 \mathrm{~B}^{2}}\right)\left(\frac{1}{\lambda_{1}^{4}}\right) \mathrm{K}^{2}, \\
\mathrm{c}_{11}=-1-\left(\frac{\mathrm{y}}{2 \mathrm{~B}^{2}}\right)\left(\frac{1}{\lambda_{1}^{3}}\right) \mathrm{K}^{2}, \mathrm{a}_{12}=-\left(\frac{\mu}{\rho \mathrm{D}^{2} \mathrm{~B}^{2}}\right)\left(\frac{1}{\lambda_{1}}\right)\left(\frac{1+2 \beta_{1}^{3}}{\beta_{1}^{2}}\right) \mathrm{K}^{2}, \\
\mathrm{a}_{21}=-\left(\frac{\beta_{1}}{\lambda_{1}^{2}}\right)\left[1-\mathrm{bg}_{1}\left(\frac{\beta_{1}^{3}-1}{1+2 \beta_{1}^{3}}\right)\right]+\frac{1}{4} \mathrm{~b} \kappa_{1}\left(\frac{1}{\lambda_{1}^{5}}\right)\left(\frac{\beta_{1}^{2}}{1+2 \beta_{1}^{3}}\right) \mathrm{K}^{2}, \mathrm{~b}_{21}=\left(\frac{\beta_{1}}{\lambda_{1}}\right)\left[1-\mathrm{bg}_{1}\left(\frac{\beta_{1}^{3}-1}{1+2 \beta_{1}^{3}}\right)\right], \\
\mathrm{a}_{22}=\left(\frac{1}{\lambda_{1}}\right)\left[1-\mathrm{bg}_{1}\left(\frac{\beta_{1}^{3}-1}{1+2 \beta_{1}^{3}}\right)-\mathrm{bg}_{1}\left\{\frac{9 \beta_{1}^{3}}{\left(1+2 \beta_{1}^{3}\right)^{2}}\right\}-2 \mathrm{~b} \kappa_{1}\left(\frac{\beta_{1}}{\beta_{1}^{3}-1}\right)\right], \mathrm{b}_{22}=-1, \\
\mathrm{a}_{23}=2 \mathrm{~b}\left(\frac{1}{\lambda_{1}}\right)\left(\frac{\beta_{1}^{2}}{1+2 \beta_{1}^{3}}\right), \mathrm{a}_{31}=-\mathrm{mb}^{2}\left(\frac{1}{\lambda_{1}^{2}}\right) \mathrm{g}_{1}\left(\kappa_{\mathrm{s}}-\kappa_{1}\right)-\frac{1}{4} \mathrm{mb}\left(\frac{1}{\lambda_{1}^{5}}\right)\left(\frac{\beta_{1}}{\beta_{1}^{3}-1}\right) \kappa_{1}\left(\kappa_{\mathrm{s}}-\kappa_{1}\right) \mathrm{K}^{2}, \\
\mathrm{~b}_{31}=\mathrm{mb}_{33}\left(\frac{1}{\lambda_{1}}\right) \mathrm{g}_{1}\left(\kappa_{\mathrm{s}}-\kappa_{1}\right), \mathrm{a}_{32}=2 \mathrm{mb}\left(\frac{1}{\lambda_{1}}\right)\left[\frac{1+2 \beta_{1}^{3}}{\left(\beta_{1}^{3}-1\right)^{2}}\right] \kappa_{1}\left(\kappa_{\mathrm{s}}-\kappa_{1}\right),
\end{gathered}
$$

Now, using (56), the coefficients in (44) are given by

$$
\begin{gathered}
\mathrm{a}_{0}=-\mathrm{a}_{23}\left(\mathrm{a}_{11} \mathrm{a}_{32}-\mathrm{a}_{12} \mathrm{a}_{31}\right)+\mathrm{a}_{33}\left(\mathrm{a}_{11} \mathrm{a}_{22}-\mathrm{a}_{12} \mathrm{a}_{21}\right), \\
\mathrm{a}_{1}=-\mathrm{a}_{23}\left(-\mathrm{a}_{12} \mathrm{~b}_{31}+\mathrm{a}_{32} \mathrm{~b}_{11}\right)+\mathrm{a}_{33}\left(\mathrm{a}_{11} \mathrm{~b}_{22}-\mathrm{a}_{12} \mathrm{~b}_{21}+\mathrm{a}_{22} \mathrm{~b}_{11}\right)+\mathrm{b}_{33}\left(\mathrm{a}_{11} \mathrm{a}_{22}-\mathrm{a}_{12} \mathrm{a}_{21}\right),
\end{gathered}
$$




$$
\begin{gathered}
a_{2}=-a_{23} c_{11} a_{32}+a_{33}\left(a_{22} c_{11}+b_{11} b_{22}\right)+b_{33}\left(a_{11} b_{22}-a_{12} b_{21}+a_{22} b_{11}\right), \\
a_{3}=a_{33} c_{11} b_{22}+b_{33}\left(a_{22} c_{11}+b_{11} b_{22}\right), a_{4}=b_{22} b_{33} c_{11},
\end{gathered}
$$

For the case of no hardening, the characteristic equation of (46) reduces to (47) with the coefficients

$$
\begin{gathered}
a_{0}=a_{11} a_{22}-a_{12} a_{21}, a_{1}=a_{11} b_{11}-a_{12} b_{21}+a_{22} b_{11}, \\
a_{2}=a_{22} c_{11}+b_{11} b_{22}, a_{3}=b_{22} c_{11} .
\end{gathered}
$$




\begin{tabular}{|c|c|}
\hline $\mathrm{b}$ & $\kappa_{\mathrm{s}}$ \\
\hline 125 & 0.000921 \\
\hline 500 & 0.01 \\
\hline 2500 & 0.012421 \\
\hline
\end{tabular}

Table 1 Values of $\left\{b, \kappa_{\mathrm{s}}\right\}$ determined by (52) which cause the same saturated value

$$
\varepsilon_{\mathrm{es}} \text { of } \varepsilon_{\mathrm{e}} \text {. }
$$




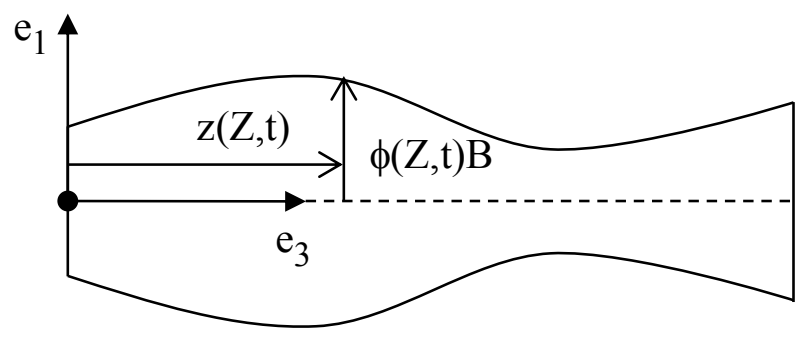

Fig. 1 Sketch of a Cosserat rod with a straight centerline and a circular cross-section having undeformed radius $B$. 

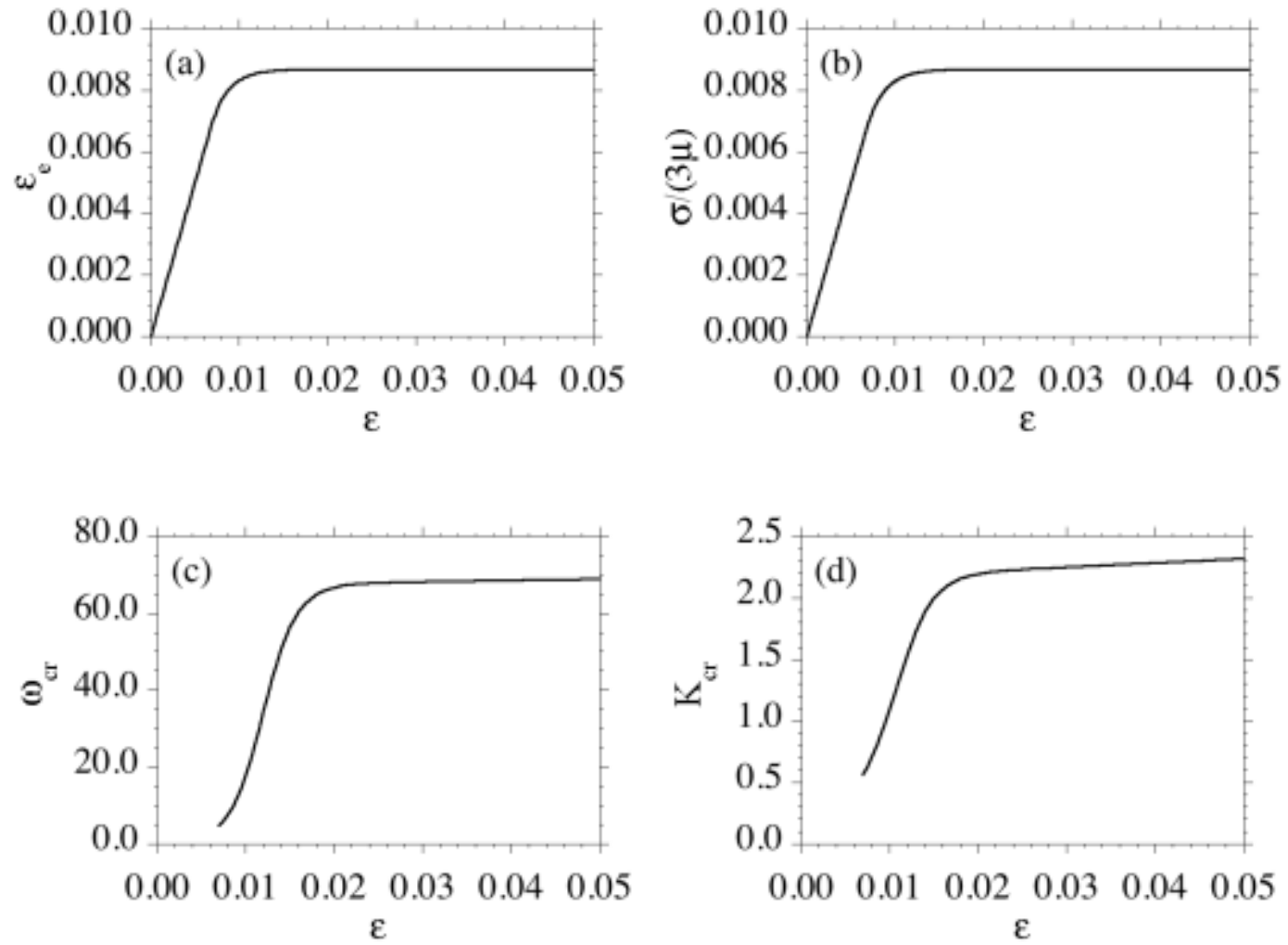

Fig. 2. Response of the reference material for no hardening: (a) elastic strain $\varepsilon_{\mathrm{e}}$; (b) axial stress $\sigma$; (c) critical growth rate $\omega_{\mathrm{cr}}$; and (d) critical wave number $\mathrm{K}_{\mathrm{cr}}$; versus nominal total strain $\varepsilon$ for $\dot{\varepsilon}=10000[1 / \mathrm{s}], \mathrm{b}=500, \kappa=0.01$ and $\mathrm{m}=0$. 


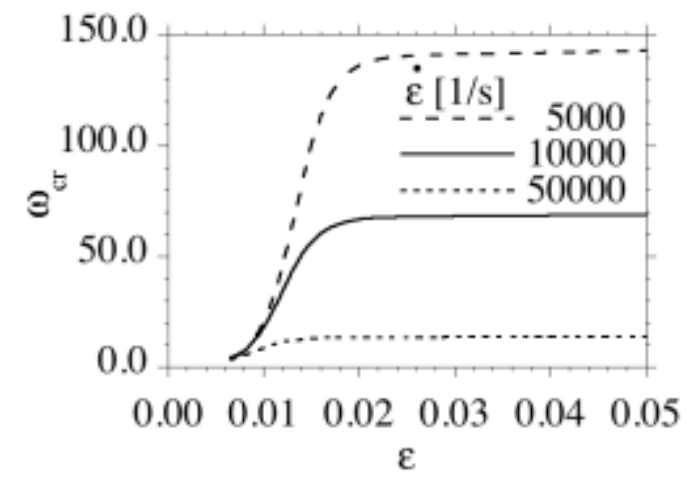

Fig. 3. Influence of the nominal total strain rate $\dot{\varepsilon}$ for no hardening: Critical growth rate $\omega_{\mathrm{cr}}$ versus nominal total strain $\varepsilon$ for $\mathrm{b}=500, \kappa=0.01, \mathrm{~m}=0$ and three strain rates $\dot{\varepsilon}$. 

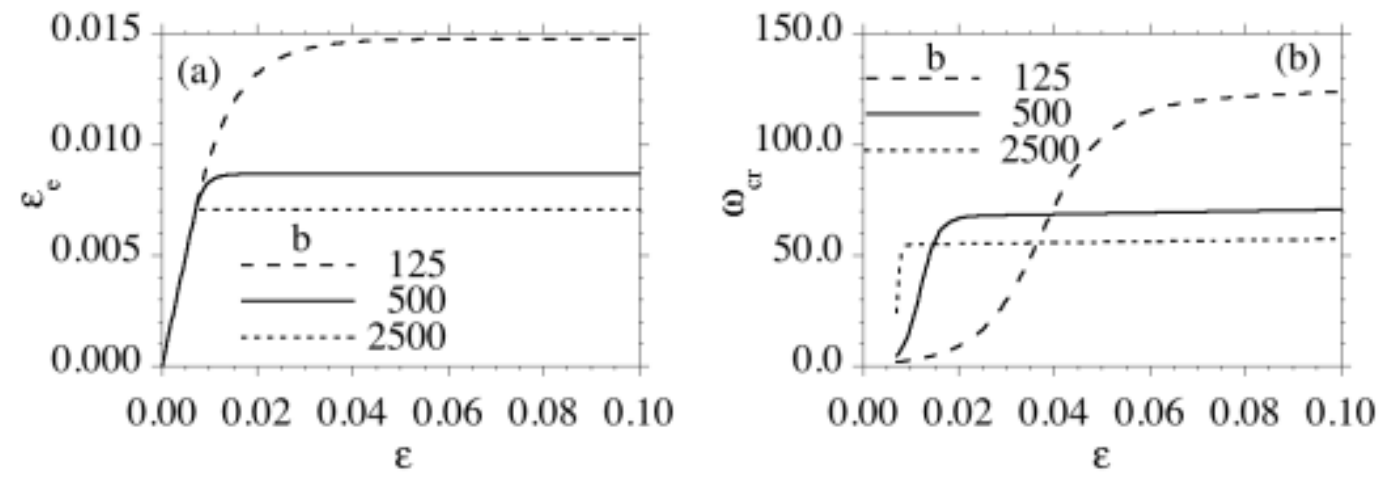

Fig. 4. Influence of the overstress constant $b$ for no hardening with different saturated values $\varepsilon_{\text {es }}$ of $\varepsilon_{\mathrm{e}}$ : (a) elastic strain $\varepsilon_{\mathrm{e}}$; (b) critical growth rate $\omega_{\mathrm{cr}}$; versus nominal total strain $\varepsilon$ for $\dot{\varepsilon}=10000[1 / \mathrm{s}], \kappa=0.01, \mathrm{~m}=0$ and three values of $\mathrm{b}$. 

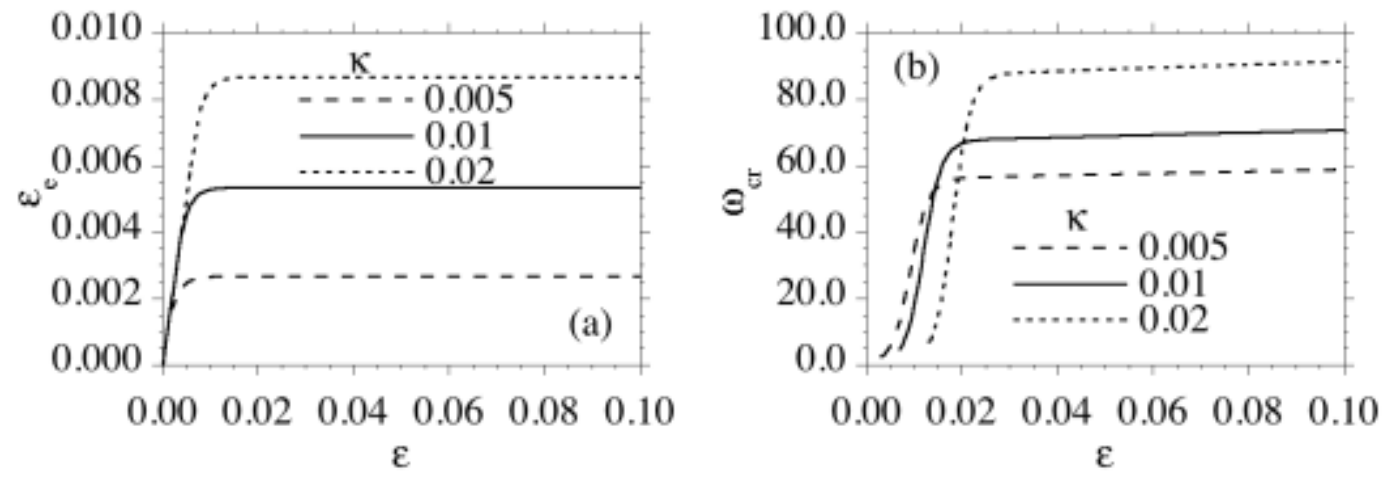

Fig. 5. Influence of $\kappa$ for no hardening: (a) elastic strain $\varepsilon_{\mathrm{e}}$; (b) critical growth rate $\omega_{\mathrm{cr}}$; versus nominal total strain $\varepsilon$ for $\dot{\varepsilon}=10000[1 / \mathrm{s}], \mathrm{b}=500, \mathrm{~m}=0$ and three values of $\kappa$. 

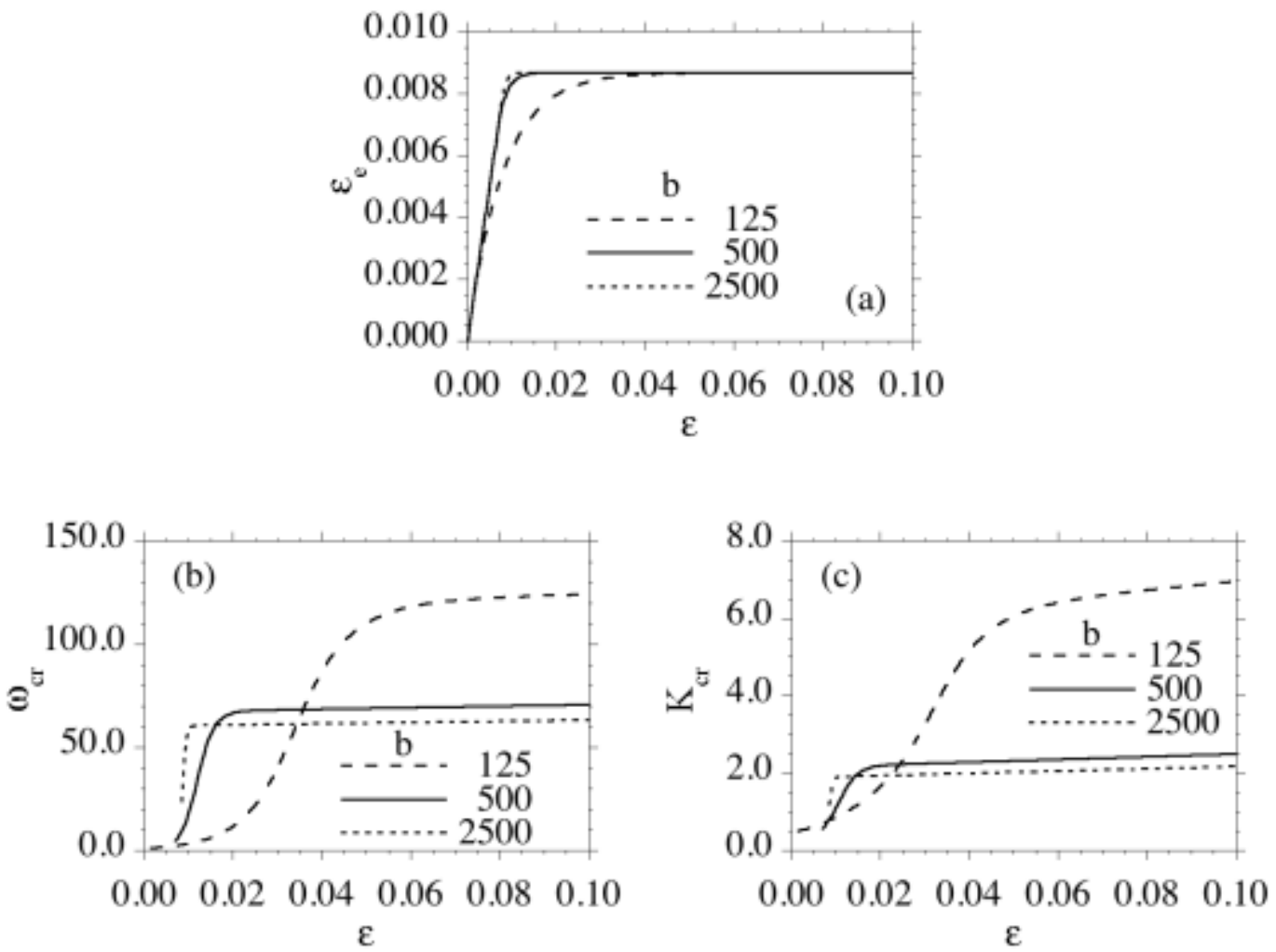

Fig. 6. Influence of the overstress constant $b$ for no hardening with the same saturated value $\varepsilon_{\mathrm{es}}$ of $\varepsilon_{\mathrm{e}}$; (a) elastic strain $\varepsilon_{\mathrm{e}}$; (b) critical growth rate $\omega_{\mathrm{cr}}$; (c) critical wave number $\mathrm{K}_{\mathrm{cr}}$; versus the nominal total strain $\varepsilon$ for $\dot{\varepsilon}=10000[1 / \mathrm{s}], \mathrm{m}=0$ and three combinations of $\left\{b, \kappa_{\mathrm{s}}\right\}$ given by $\{125,0.000921\},\{500,0.01\},\{2500,0.012421\}$. 

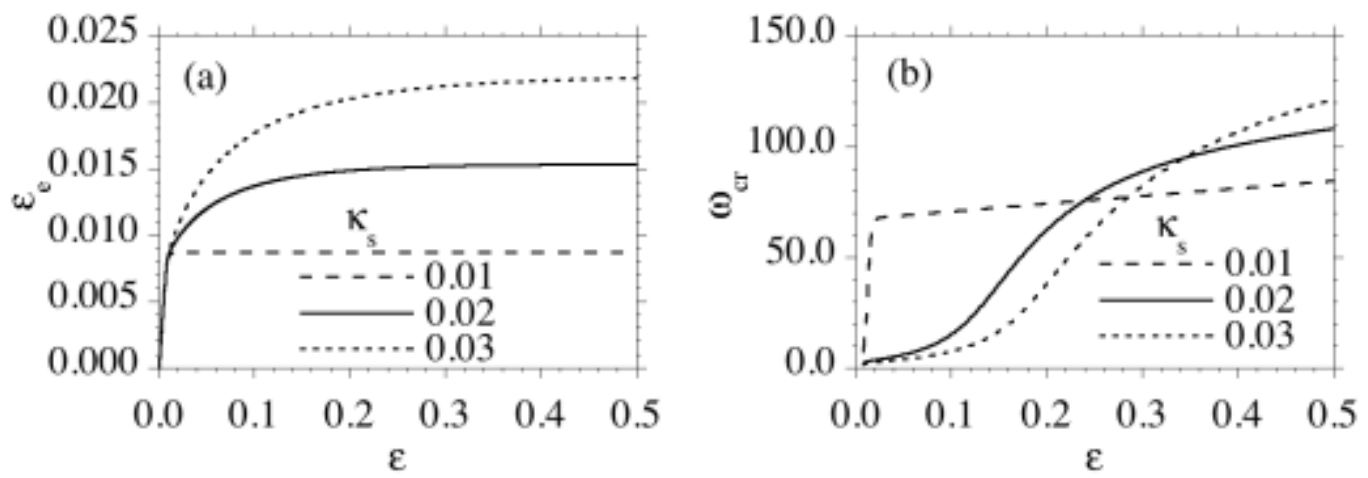

Fig. 7. Influence of the saturated value $\kappa_{\mathrm{S}}$ of hardening: (a) elastic strain $\varepsilon_{\mathrm{e}}$; (b) critical growth rate $\omega_{\text {cr }}$; versus the nominal total strain $\varepsilon$ for $\dot{\varepsilon}=10000[1 / \mathrm{s}], \mathrm{b}=500, \kappa_{0}=0.01$, $\mathrm{m}=0.2$ and three values of $\kappa_{\mathrm{S}}(0.01,0.02,0.03)$ 

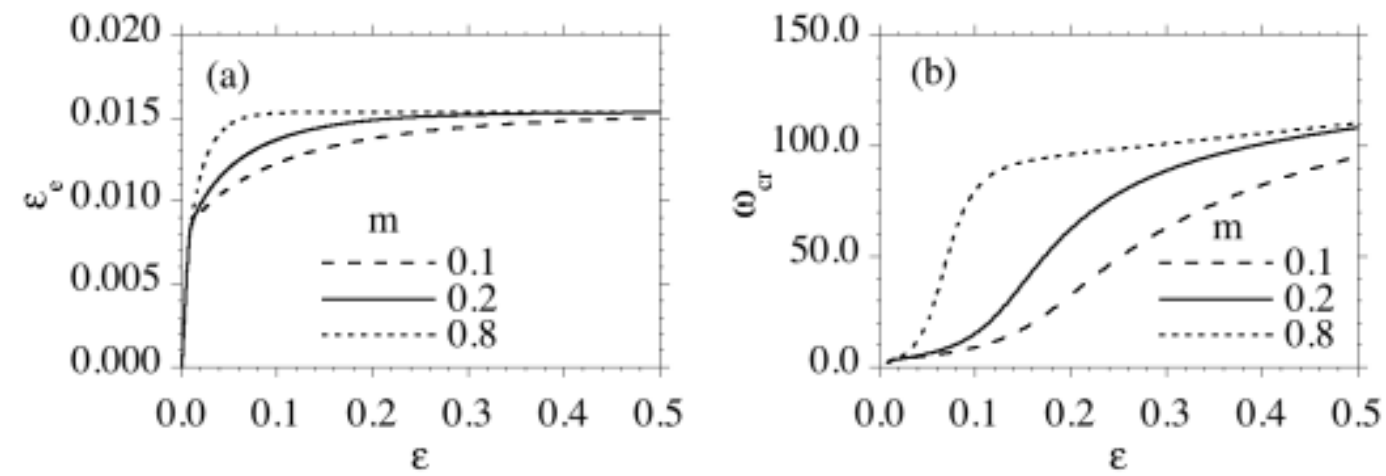

Fig. 8. Influence of the hardening rate $\mathrm{m}$ : (a) elastic strain $\varepsilon_{\mathrm{e}}$; (b) critical growth rate $\omega_{\mathrm{cr}}$; versus the nominal total strain $\varepsilon$ for $\dot{\varepsilon}=10000[1 / \mathrm{s}], \mathrm{b}=500, \kappa_{0}=0.01, \kappa_{\mathrm{s}}=0.02$ and three values of $\mathrm{m}(0.1,0.2,0.8)$. 

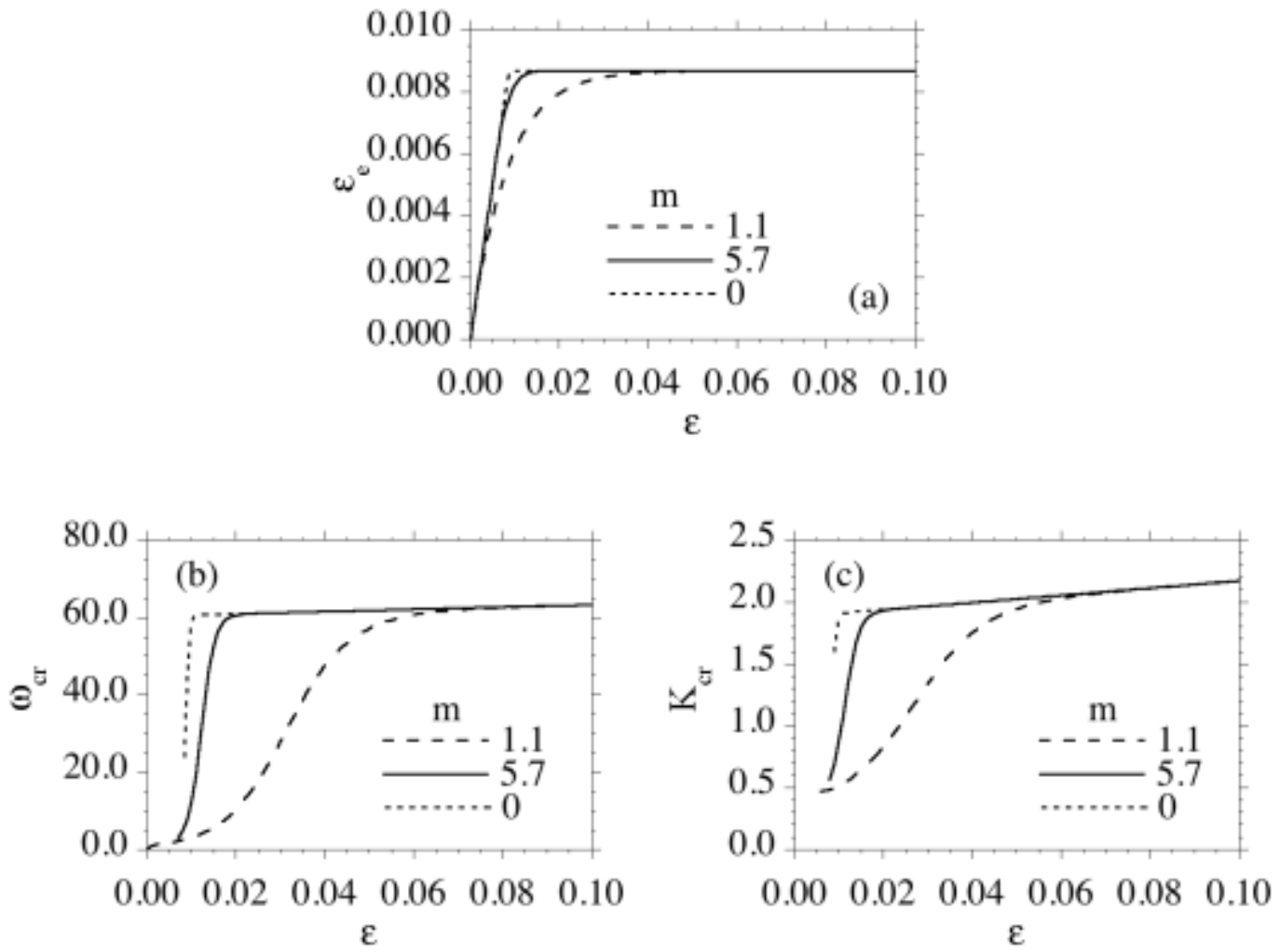

Fig. 9. Influence of the hardening rate $\mathrm{m}$ for a small overstress with the same saturated value $\varepsilon_{\mathrm{es}}$ of $\varepsilon_{\mathrm{e}}$ : (a) elastic strain $\varepsilon_{\mathrm{e}}$; (b) critical growth rate $\omega_{\mathrm{cr}}$; and (c) critical wave number $\mathrm{K}_{\mathrm{cr}}$; versus the nominal total strain $\varepsilon$ for $\dot{\varepsilon}=10000[1 / \mathrm{s}], \mathrm{b}=2500$, $\kappa_{\mathrm{s}}=0.012421$ with Case $1\left\{\kappa_{0}=0.000921, \mathrm{~m}=1.1\right\}$, Case $2\left\{\kappa_{0}=0.01, \mathrm{~m}=5.7\right\}$ and Case $3\left\{\kappa_{0}=\kappa_{\mathrm{s}}, \mathrm{m}=0\right.$, no hardening $\}$. 

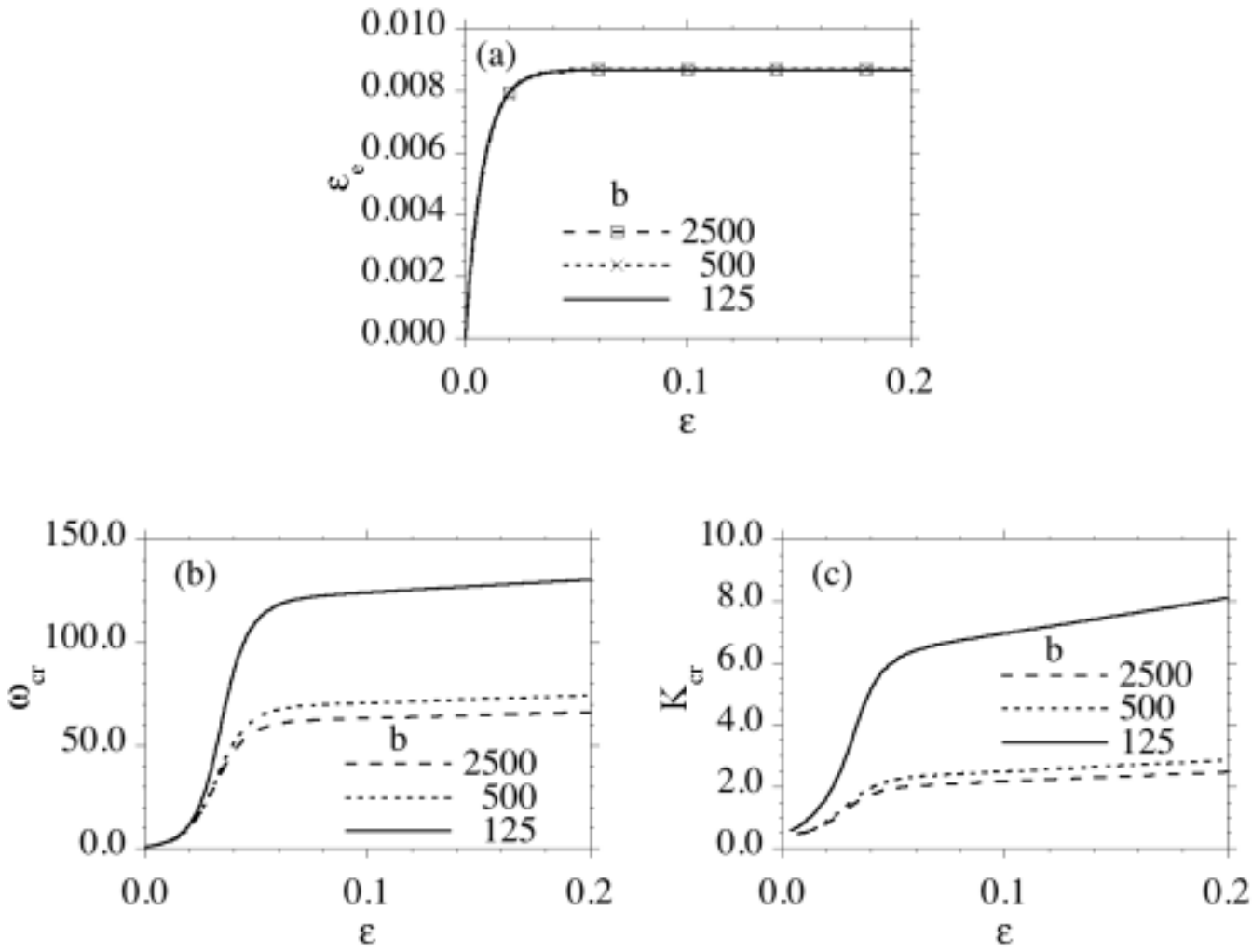

Fig. 10. Influence of the overstress constant $b$ with the same loading curve: (a) elastic strain $\varepsilon_{\mathrm{e}}$; (b) critical growth rate $\omega_{\mathrm{cr}}$; and (c) critical wave number $\mathrm{K}_{\mathrm{cr}}$; versus the nominal total strain $\varepsilon$ for $\dot{\varepsilon}=10000[1 / \mathrm{s}], \kappa_{0}=0.000921$ with

Case $1\left\{b=2500, \kappa_{\mathrm{s}}=0.012421, \mathrm{~m}=1.1\right\}$, Case $2\left\{\mathrm{~b}=500, \kappa_{\mathrm{S}}=0.01, \mathrm{~m}=1.1\right\}$ and Case $3\left\{b=125, \kappa_{0}=\kappa_{\mathrm{S}}\right.$, no hardening $\}$. 

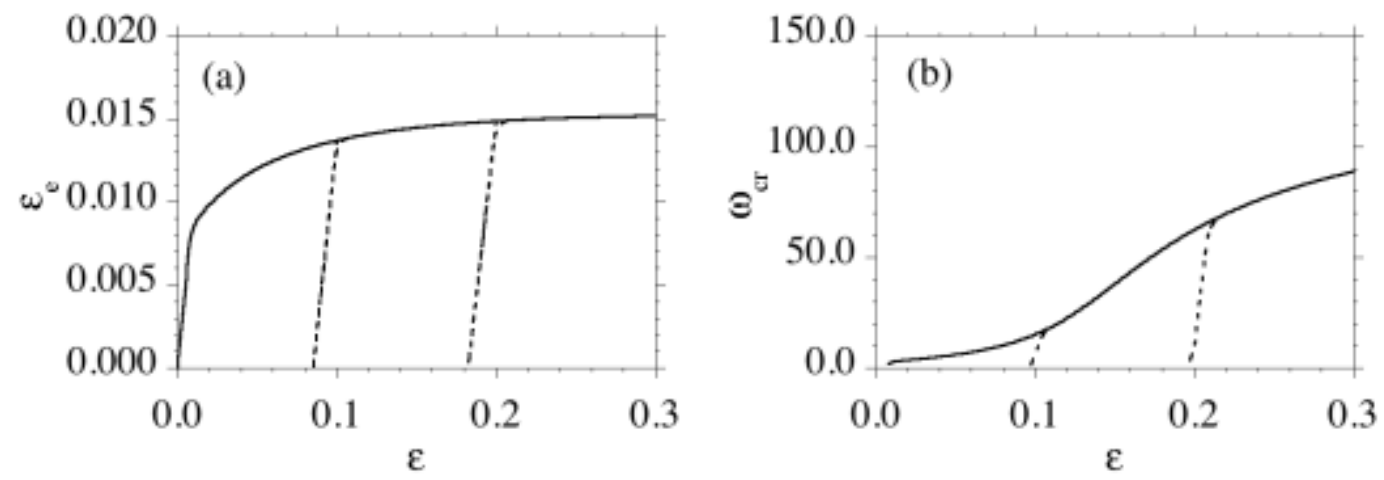

Fig. 11. Loading/unloading/reloading response for a small overstress: (a) elastic strain $\varepsilon_{\mathrm{e}}$;

(b) critical growth rate $\omega_{\text {cr }}$; versus the nominal total strain $\varepsilon$ for $\dot{\varepsilon}=10000[1 / \mathrm{s}], \mathrm{b}=500$, $\kappa_{0}=0.01, \kappa_{\mathrm{s}}=0.02$ and $\mathrm{m}=0.2$. The continuous loading case is compared with a loading process with two unloading/reloading portions. 

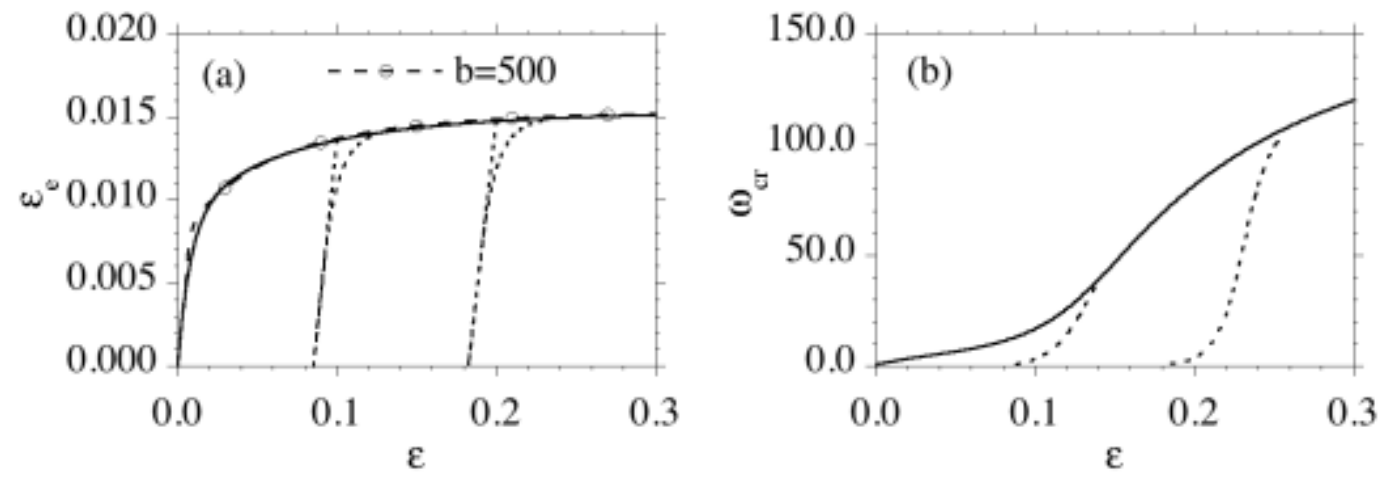

Fig. 12. Loading/unloading/reloading response for a large overstress: (a) elastic strain $\varepsilon_{\mathrm{e}}$;

(b) critical growth rate $\omega_{\mathrm{cr}}$; versus the nominal total strain $\varepsilon$ for $\dot{\varepsilon}=10000[1 / \mathrm{s}], \mathrm{b}=125$, $\kappa_{0}=0.00260, \kappa_{\mathrm{s}}=0.010860$ and $\mathrm{m}=0.17$. Fig. 12a includes the response in Fig. $11 \mathrm{a}(\mathrm{b}$ $=500$ ) for comparison. The continuous loading case is compared with a loading process with two unloading/reloading portions. 